\title{
Real Time Traffic Control under Time Varying Incoming Flow Rates of Vehicles to Minimize Waiting Time of Vehicles at Road Arterial Networks
}

\author{
Yogeswary Raviraj \\ Department of Mathematical Sciences \\ Faculty of Applied Sciences \\ South Eastern University of Sri Lanka \\ Sri Lanka \\ W. B. Daundasekera \\ Department of Mathematics \\ Faculty of Science \\ University of Peradeniya, Sri Lanka
}

\begin{abstract}
This paper presents two real time nonlinear quadratic programming models according to two special oversaturation conditions to minimize the aggregate delay time of vehicles at each intersection by minimizing the total number of vehicles at each intersection at the signalized two intersections arterial. This can be extended to more than two intersections arterial network by expanding the groups which are explained in this paper. These models are developed under time varying incoming flow rates of vehicles. The most important factor of traffic signal control is the number of vehicles in a queue on the lanes of intersections. The initial number of vehicles on each lane at the intersections is counted by a camera which is the most exact method among other existing methods. These models are developed to minimize the number of waiting vehicles from cycle to cycle. These proposed models include inter green signal time this is one of the main aspect compared to other existing models proposed in the former research. These models also include restriction for upper bound for cycle time allocation which leads to exact and appropriate allocation for green signal time. Non- negative queue lengths on lanes are maintained under special conditions for oversaturation case. The lower bound of green signal time is attained by oversaturation conditions of the models. These proposed models are solved by the interior point algorithm method coded in MATLAB environment.
\end{abstract}

Keywords: traffic signals; arterial networks; number of vehicles; aggregate delay time; nonlinear optimization; interior point algorithm

\section{Introduction}

The monitoring and controlling of traffic at roads is a major concern in many countries, because number of vehicles on the road increases daily that leads to traffic congestion problem. Traffic congestion wastes a huge amount of countries' national income for fuel and creates traffic - related environmental and socio economic problems. Traffic signals are the most suitable method of controlling traffic in busy intersections. A coordinated arterial signal system consists of two or more traffic signals having a fixed time relationship to each other. The relationship among arterial signals may be designed to permit travel without stopping or progression. If the system is operated without coordination, progression is not possible, and increase stops and delays. Fuel consumption increases with delays and stops.

Some existing optimization models to overcome the traffic control problem at road arterial networks are: Sydney Coordinated Adaptive Traffic System (SCATS)(Zong Tian et al. 2011), Optimization Policies for Adaptive Control (OPAC)(Gartner, N H. 1983, Zhili Tian 2002), Urban Traffic Optimization by Integrated Automation (UTOPIA)(Yuanchang XIE 2007), PRODYN(Yuanchang XIE 2007), Split Cycle Offset Optimization Technique (SCOOT)(SCOOT User Guide 2003), Adaptive Limited Look - ahead Optimization of Network Signals Decentralized (ALLONS - D)(Yuanchang XIE 2007).Delay (Tang-Hsien Chang et al. 2000) and the number of vehicles waiting are the important measures of effectiveness for signalized intersections. This research considers the oversaturation level at road intersections, where the intersections considered have four signals and the green time is allocated for all four signals. Our objective of this research is to formulate mathematical models to minimize aggregate delay (AKCELIK, R. 1980) time of vehicles and the total number of vehicles which are waiting on the lanes of a road intersection due to red signal by allocating sufficient amount of green time for each signal and cycle time. 
To maintain the feasibility, the upper bound for cycle time and number of vehicles waiting are restricted. The real time data is calculated by using cameras (Mrs. P M Daigavane et al. 2010) installed on every lane at the road intersections. Those models are solved by interior point algorithm coded in MATLAB environment (Ahmet Yazici et al. 2008).

\section{Methodology}

\subsection{Arterial Network Design}

A road consist of two intersections arterial network is considered (Aboudolas, K. et al 2009). These intersections are $i$ and $j$, and link of intersection is $z$. For each signalized intersection $m$ the set of incoming $I_{m}$ and outgoing $O_{m}$ links, where $m=i, j$. Each intersection makes its own plan with a difference of offset time. In our models the offset time is zero. Assume that cycle time of intersection $i, C_{i}$ and cycle time of intersection $j, C_{j}$ are same and equal to $C$. The signal control plan of intersection $m$ is based on a fixed number of stages that belong to the set $F_{m}$, while $v_{z}$ denotes the set of stages where link $z$ has right of way. The saturation flow $s_{z}$ of link $z$ and the turning rates $t_{w, z}$, where $w \in I_{m}$ and $z \in$ $O_{m}$, are assumed to be known and constant or may be time varying.

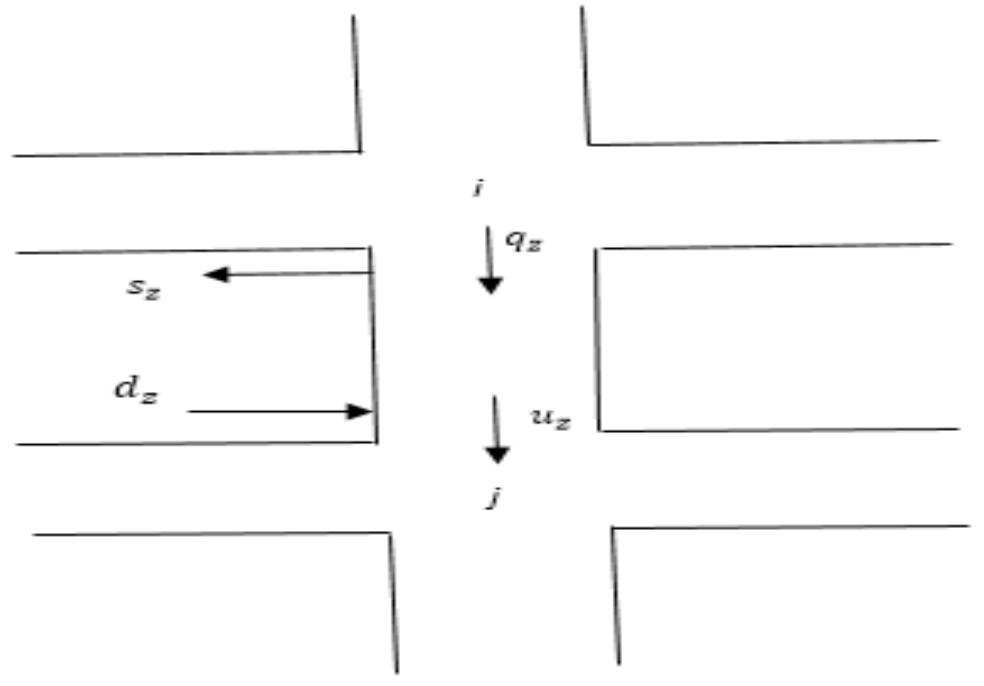

Figure 1: A link of two intersections

Each intersection consists of four lanes. $g_{m l}$ is the green time of stage $l$ at intersection $m$. Green time constraint is given by $\sum_{l \in F_{m}} g_{m l}=C, m=i, j$.

Green time control conditions are:

$g_{m l} \geq\left(g_{m l}\right)_{\min }, m=i, j$, where $\left(g_{m l}\right)_{\min }$ is the minimum permissible green time for stage $l$ at intersection, $m=i, j$.

Consider a link $z$ connecting two intersections $i$ and $j$ such that $z \in O_{i}$ and $z \in I_{j}$ as shown in Figure 1 . The dynamics of link $z$ are given by the conservation equation $x_{z}(k+1)=x_{z}(k)+T\left[q_{z}(k)-s_{z}(k)+d_{z}(k)-u_{z}(k)\right]$,

where $x_{z}(k)$ is the number of vehicles within link $z$ (or queue length) at time $k T, q_{z}(k)$ and $u_{z}(k)$ are the inflow and outflow, respectively, of link $z$ in the sample period $[k T,(k+1) T] ; T$ is the discrete-time step and $k=0,1, \ldots$ the discrete-time index; $d_{z}$ and $s_{z}$ are the demand and the exit flow within the link, respectively. For the exit flow

$s_{z}(k)=t_{z, 0} q_{z}$, where the exit rates $t_{z, 0}$ are assumed to be known. The inflow to the link $z$ is given by $q_{z}(k)=$ $\sum_{w \in I_{i}} t_{w, z} u_{w}(k)$, where $t_{w, z}$ with $w \in I_{i}$ are the turning rates towards link $z$ from the links that enter intersection $i$.

Queue lengths are subject to the constraints

$0 \leq x_{z}(k) \leq x_{z, \max }$,

where $x_{z, \max }$ is the maximum admissible queue length. These constraints may automatically lead to a suitable upstream gating in order to protect downstream areas from oversaturation during periods of high demand.

The outflow (real flow) $u_{z}$ of link $z$ is equal to the saturation flow $s_{z}$. If the link has right of way, and equal to zero otherwise. However, if the discrete-time step $T$ is equal to $C$, an average value for each period (modeled flow) is obtained by

$u_{z}(k)=G_{z}(k) s_{z} /_{C}$,

where $G_{z}$ is the green time of link $z$, calculated as $G_{z}(k)=\sum_{w \in v_{z}} g_{j, w}(k)$. 


\subsection{Phase Sequence Design}

In this research, we consider a signalized arterial road with two intersections namely Intersection 1 and Intersection 2 and eight lanes namely Lane $i j, i=1,2$ and $j=1,2,3,4$. We divide this arterial into four stages: in Stage 1, green signals (same amount of green signal time) will be on for Lane 12 and Lane 22 by grouping Lane 12 and Lane 22 ([Lane 12Lane 22]), where the vehicles which are waiting on the Lane 12 and Lane 22 can go to each other three lanes through the intersection as shown in Stage 1 in the below Figure 2. Similarly, when the green signals are on for other two grouped lanes ([Lane 14-Lane 24], [Lane 11- Lane 21], [Lane 13-Lane 23]) the vehicles waiting on those lanes will proceed to different lanes as described in Stage 2, Stage 3 and Stage 4 in the Figure 2 below:

Stage 1
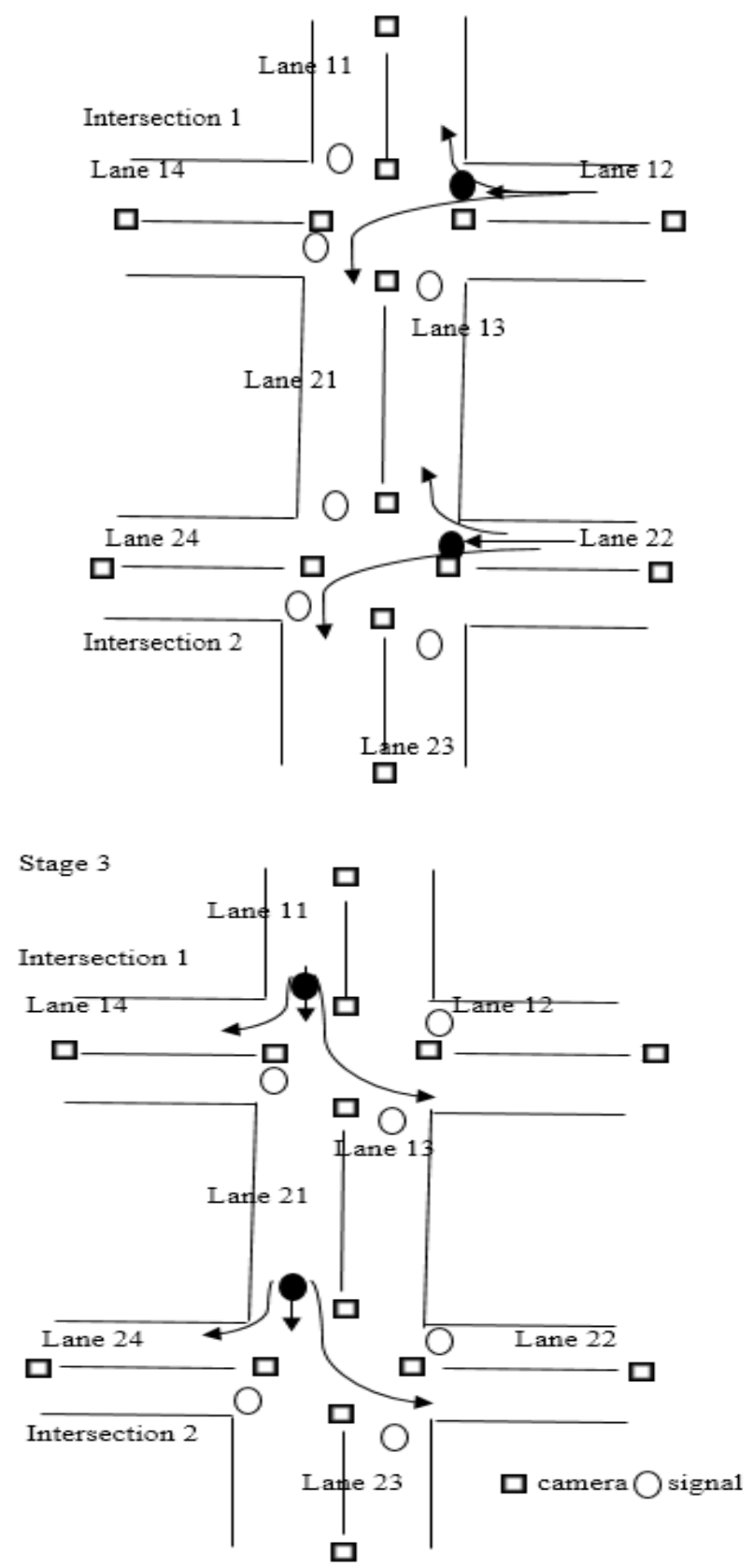

Stage 2
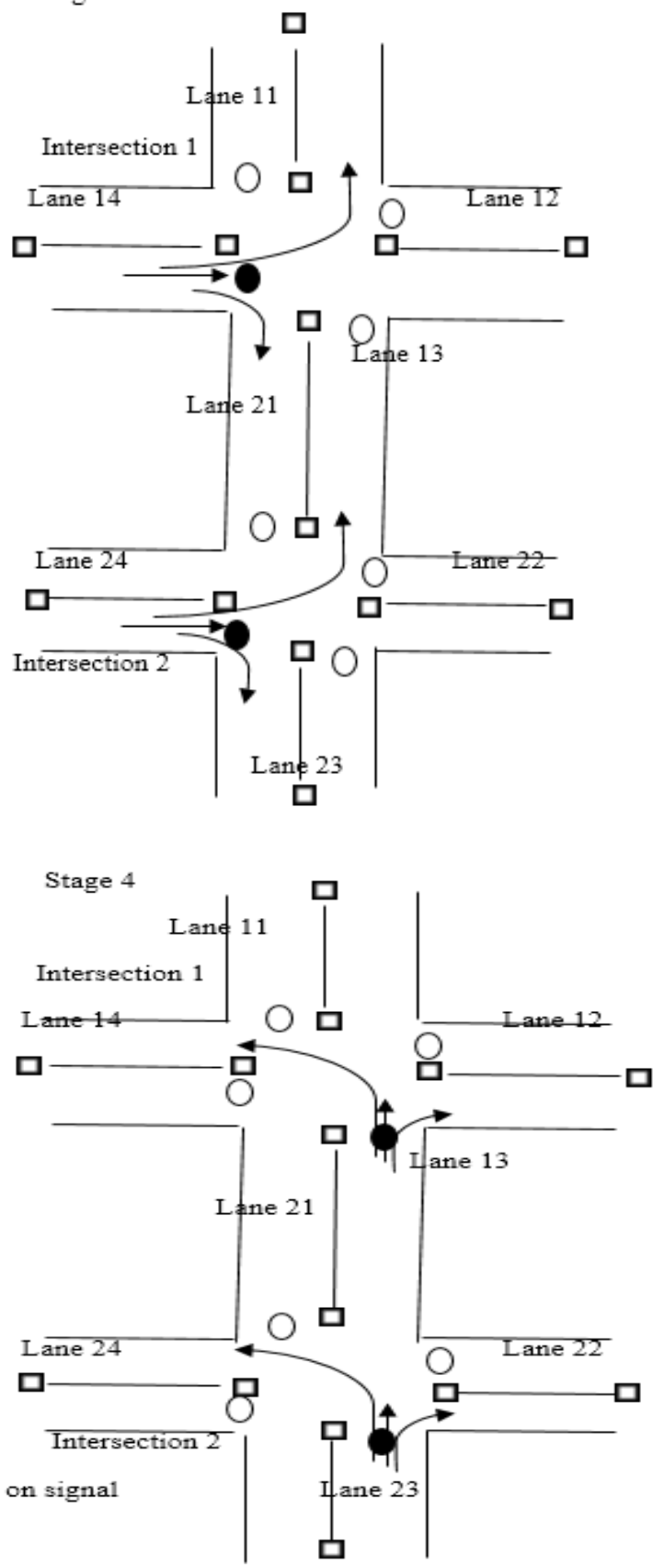

Figure 2: Green signals and stages (phases).

Each intersection makes its own plan with a difference of offset time. In this model the offset time for cycles is set to zero. Vehicles entering into the Lane 13 will be during green signal on for Lane 12, Lane 13 and Lane 14 only. Similarly, vehicles entering into the Lane 21 will be during green signal on for Lane 21, Lane 22 and Lane 24 only. 


\subsection{Formulation of the Model:}

The notations used in this model are:

Lane $i j: j^{\text {th }}$ lane of $i^{\text {th }}$ intersection at the arterial road $i=1,2, j=1,2,3,4$,

$N_{i j}(k)$ : Number of vehicles on Lane $j$ of $i^{\text {th }}$ intersection at the beginning of cycle $k, i=1,2, j=1,2,3,4$,

$N T_{i}(k)$ : Total number of vehicles at intersection $i$ at the beginning of cycle $k, i=1,2$,

$I g_{i j}(k)$ : Inter green time for the signal of Lane $j$ of $i^{\text {th }}$ intersection during cycle $k, i=1,2, j=1,2,3,4$,

$g_{i j}(k)$ : Allocated green time for the signal of Lane $j$ of $i^{\text {th }}$ intersection during cycle $k, i=1,2, j=1,2,3,4$,

$f_{i j}(k)$ : In coming flow rate of vehicles for the Lane $j$ of $i^{\text {th }}$ intersection during cycle $k$ and maximum cycle time, $i=1,2$, $j=1,2,3,4$,

$f_{i j}^{\left(g_{i m}(k)+\operatorname{Ig} g_{i m}(k)\right)}:$ In coming flow rate of vehicles for the Lane $j$ of $i^{\text {th }}$ intersection $i=1,2, j=1,2,3,4$ during cycle $k$ and allocated green time and inter green time for the signal for Lane $m, m=1,2,3,4$,

Here, $f_{i j}^{\left(g_{i m}(k)+I g_{i m}(k)\right)}=\frac{f_{i j}(k)\left(g_{i m}(k)+I g_{i m}(k)\right)}{\left(C T_{i}\right)_{\max }}$.

$s_{i j}(k)$ : Outgoing flow rate of vehicles for the Lane $j$ of $i^{\text {th }}$ intersection during cycle $k, i=1,2, j=1,2,3,4$,

$S_{13}(k)$ : Saturation flow rate of vehicles for Lane 13 during cycle $k$,

$S_{21}(k)$ : Saturation flow rate of vehicles for Lane 21 during cycle $k$,

$t_{13}(k)$ : Average turning rates of vehicles for Lane 13 during cycle $k$,

$t_{21}(k)$ : Average turning rates of vehicles for Lane 21 during cycle $k$,

$e_{13}(k)$ : Exit flow of vehicles for Lane 13 during cycle $k$,

$\mathrm{e}_{21}(k)$ : Exit flow of vehicles for Lane 21 during cycle $k$,

$d_{13}(k)$ : Demand flow of vehicles for Lane 13 during cycle $k$,

$\mathrm{d}_{21}(k)$ : Demand flow of vehicles for Lane 21 during cycle $k$,

$\left(g_{i j}(k)\right)_{\min }$ : Minimum green time for the signal for Lane $j$ of $i^{\text {th }}$ intersection during cycle $k, i=1,2, j=1,2,3,4$,

$\left(g_{i j}(k)\right)_{\max }$ : Maximum green time for the signal for Lane $j$ of $i^{\text {th }}$ intersection during cycle $k, i=1,2, j=1,2,3,4$,

$C_{i}(k)$ : Cycle time of intersection $i$ during cycle $k, i=1,2$,

$\left(C T_{i}\right)_{\min }$ : Minimum cycle time of $i^{\text {th }}$ intersection $i=1,2$,

$\left(C T_{i}\right)_{\max }$ : Maximum cycle time of $i^{\text {th }}$ intersection $i=1,2$,

$t_{1 j}(k)$ : Average vehicle turning rates of Intersection 1 for Lane $j$ during cycle $k, j=1,2,4$,

$t_{2 j}(k)$ : Average vehicle turning rates of Intersection 2 for Lane $j$ during cycle $k, j=2,3,4$,

$D_{i j}(k+1)$ : Aggregate delay time of vehicles at the end of cycle $k$ for Lane $j$ of $i^{\text {th }}$ intersection $i=1,2, j=1,2,3,4$.

$D T_{i}(k+1)$ : Aggregate delay time of vehicles at the end of cycle $k$ for intersection $i, i=1,2$

The oversaturation condition for a particular lane is that the outgoing number of vehicles during green signal should be strictly less than the total of the number of existing vehicles at the beginning of the cycle and incoming number of vehicles during the previous stages green signal time and inter green time and incoming number of vehicles during green signal time.

The number of vehicles at oversaturated situation and aggregate delay time of four stages at Intersection 1 are illustrated (modified from (Tang-Hsien Chang et al. 2000)) in the Figure 3: 
Intersection 1

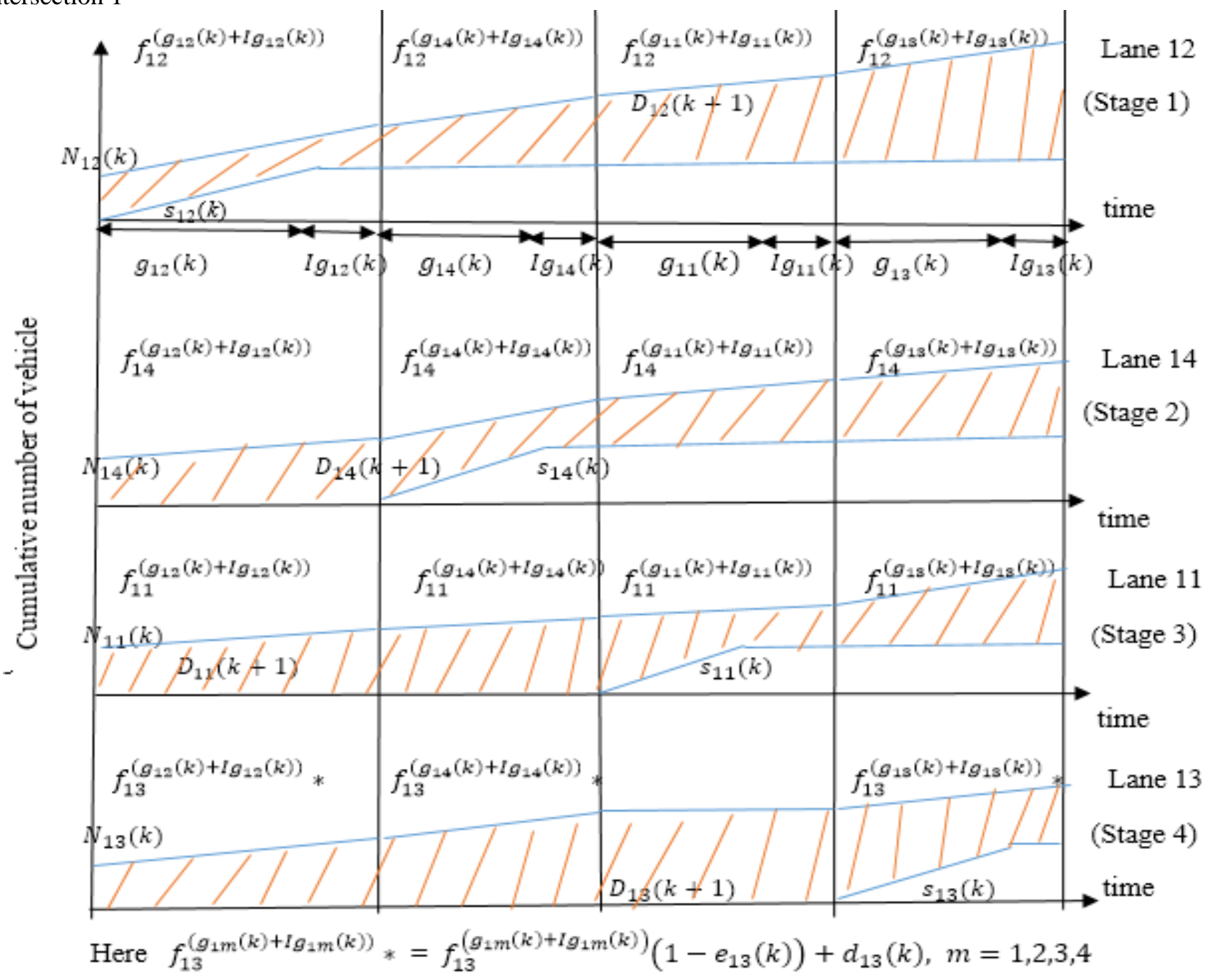

Figure 3: Number of vehicle and delay of four stages at Intersection 1.

The inequalities (Peng CHEN et al. 2013) which satisfy the oversaturation condition in each stage are given below:

$s_{12}(k) g_{12}(k)<N_{12}(k)+f_{12}^{\left(g_{12}(k)+I g_{12}(k)\right)} g_{12}(k)$,

$s_{14}(k) g_{14}(k)<N_{14}(k)+f_{14}^{\left(g_{12}(k)+I g_{12}(k)\right)}\left(g_{12}(k)+I g_{12}(k)\right)+f_{14}^{\left(g_{14}(k)+I g_{14}(k)\right)} g_{14}(k)$,

$s_{11}(k) g_{11}(k)<N_{11}(k)+f_{11}^{\left(g_{12}(k)+I g_{12}(k)\right)}\left(g_{12}(k)+I g_{12}(k)\right)+f_{11}^{\left(g_{14}(k)+I g_{14}(k)\right)}\left(g_{14}(k)+I g_{14}(k)\right)+$

$f_{11}^{\left(g_{11}(k)+I g_{11}(k)\right)} g_{11}(k)$,

$s_{13}(k) g_{13}(k)<N_{13}(k)+\left(1-e_{13}(k)\right)\left(f_{13}^{\left(g_{12}(k)+I g_{12}(k)\right)}\left(g_{12}(k)+I g_{12}(k)\right)+f_{13}^{\left(g_{14}(k)+I g_{14}(k)\right)}\left(g_{14}(k)+I g_{14}(k)\right)+\right.$ $\left.f_{13}^{\left(g_{13}(k)+I g_{13}(k)\right)} g_{13}(k)\right)+d_{13}(k)\left(g_{12}(k)+I g_{12}(k)+g_{14}(k)+I g_{14}(k)+g_{13}(k)\right)$,

Here, $f_{1 j}^{\left(g_{1 m}(k)+I g_{1 m}(k)\right)}=\frac{f_{1 j}(k)\left(g_{1 m}(k)+I g_{1 m}(k)\right)}{\left(C T_{1}\right) \max }, j=1,2,3,4, m=1,2,3,4$,

$f_{13}(k)=t_{22}(k) s_{22}(k)+t_{23}(k) s_{23}(k)+t_{24}(k) s_{24}(k)$ and $e_{13}(k)=t_{13}(k) f_{13}(k)$.

The aggregate delay time in each stage of the model is illustrated below:

In Stage 1 Intersection 1, the aggregate delay time for $(k+1)^{\text {th }}$ cycle (i.e. aggregate delay time at the end of $k^{\text {th }}$ cycle) is calculated by the aggregate delay time of vehicles on the Lane 12 during green signal is on and after the green signal is off which is represented by the shaded area of Stage 1 Intersection 1 in Figure 3. 
In Stage 2 Intersection 1, the aggregate delay time for $(k+1)^{\text {th }}$ cycle (aggregate delay time at the end of $k^{\text {th }}$ cycle) is calculated by the aggregate delay time of vehicles on the Lane 14 before green signal is on, during green signal is on and after the green signal is off which is represented by the shaded area of Stage 2 Intersection 1 in Figure 3. The other two stages can also be illustrated in a similar manner. Aggregate delay time for vehicles in each stage at the end of cycle $\mathrm{k}$ is calculated from the area of the shaded region in the Figure 3 as given below:

$$
\begin{aligned}
& D_{12}(k+1)=N_{12}(k)\left(g_{12}(k)+I g_{12}(k)\right)+\frac{1}{2}\left(g_{12}(k)+I g_{12}(k)\right)^{2} f_{12}^{\left(t_{12}(k)+I g_{12}(k)\right)}+\left(N_{12}(k)+\right. \\
& \left.f_{12}^{\left(g_{12}(k)+I g_{12}(k)\right)}\left(g_{12}(k)+I g_{12}(k)\right)\right)\left(g_{14}(k)+I g_{14}(k)\right)+\frac{1}{2}\left(g_{14}(k)+I g_{14}(k)\right)^{2} f_{12}^{\left(g_{14}(k)+I g_{14}(k)\right)}+\left(N_{12}(k)+\right. \\
& \left.f_{12}^{\left(g_{12}(k)+I g_{12}(k)\right)}\left(g_{12}(k)+I g_{12}(k)\right)+f_{12}^{\left(g_{14}(k)+I g_{14}(k)\right)}\left(g_{14}(k)+I g_{14}(k)\right)\right)\left(g_{11}(k)+I g_{11}(k)\right)+\frac{1}{2}\left(g_{11}(k)+\right. \\
& \left.I g_{11}(k)\right)^{2} f_{12}^{\left(g_{11}(k)+I g_{11}(k)\right)}+\left(N_{12}(k)+f_{12}^{\left(g_{12}(k)+I g_{12}(k)\right)}\left(g_{12}(k)+I g_{12}(k)\right)+f_{12}^{\left(g_{14}(k)+I g_{14}(k)\right)}\left(g_{14}(k)+I g_{14}(k)\right)+\right. \\
& \left.f_{12}^{\left(g_{11}(k)+I g_{11}(k)\right)}\left(g_{11}(k)+I g_{11}(k)\right)\right)\left(g_{13}(k)+I g_{13}(k)\right)+\frac{1}{2}\left(g_{13}(k)+I g_{13}(k)\right)^{2} f_{12}^{\left(g_{13}(k)+I g_{13}(k)\right)}- \\
& \frac{1}{2} s_{12}(k)\left(g_{12}(k)\right)^{2}-s_{12}(k) g_{12}(k)\left(I g_{12}(k)+g_{14}(k)+I_{14}(k)+g_{11}(k)+I g_{11}(k)+g_{13}(k)+I g_{13}(k)\right), \\
& D_{14}(k+1)=N_{14}(k)\left(g_{12}(k)+I g_{12}(k)\right)+\frac{1}{2}\left(g_{12}(k)+I g_{12}(k)\right)^{2} f_{14}^{\left(t_{12}(k)+I g_{12}(k)\right)}+\left(N_{14}(k)+\right. \\
& \left.f_{14}^{\left(g_{12}(k)+I g_{12}(k)\right)}\left(g_{12}(k)+I g_{12}(k)\right)\right)\left(g_{14}(k)+I g_{14}(k)\right)+\frac{1}{2}\left(g_{14}(k)+I g_{14}(k)\right)^{2} f_{14}^{\left(g_{14}(k)+I g_{14}(k)\right)}+\left(N_{14}(k)+\right. \\
& \left.f_{14}^{\left(g_{12}(k)+I g_{12}(k)\right)}\left(g_{12}(k)+I g_{12}(k)\right)+f_{14}^{\left(g_{14}(k)+I g_{14}(k)\right)}\left(g_{14}(k)+I g_{14}(k)\right)\right)\left(g_{11}(k)+I g_{11}(k)\right)+\frac{1}{2}\left(g_{11}(k)+\right. \\
& \left.I g_{11}(k)\right)^{2} f_{14}^{\left(g_{11}(k)+I g_{11}(k)\right)}+\left(N_{14}(k)+f_{14}^{\left(g_{12}(k)+I g_{12}(k)\right)}\left(g_{12}(k)+I g_{12}(k)\right)+f_{14}^{\left(g_{14}(k)+I g_{14}(k)\right)}\left(g_{14}(k)+I g_{14}(k)\right)+\right. \\
& \left.f_{14}^{\left(g_{11}(k)+I g_{11}(k)\right)}\left(g_{11}(k)+I g_{11}(k)\right)\right)\left(g_{13}(k)+I g_{13}(k)\right)+\frac{1}{2}\left(g_{13}(k)+I g_{13}(k)\right)^{2} f_{14}^{\left(g_{13}(k)+I g_{13}(k)\right)}- \\
& \frac{1}{2} s_{14}(k)\left(g_{14}(k)\right)^{2}-s_{14}(k) g_{14}(k)\left(I g_{14}(k)+g_{11}(k)+I g_{11}(k)+g_{13}(k)+I g_{13}(k)\right) \text {, } \\
& D_{11}(k+1)=N_{11}(k)\left(g_{12}(k)+I g_{12}(k)\right)+\frac{1}{2}\left(g_{12}(k)+I g_{12}(k)\right)^{2} f_{11}^{\left(t_{12}(k)+I g_{12}(k)\right)}+\left(N_{11}(k)+\right. \\
& \left.f_{11}^{\left(g_{12}(k)+I g_{12}(k)\right)}\left(g_{12}(k)+I g_{12}(k)\right)\right)\left(g_{14}(k)+I g_{14}(k)\right)+\frac{1}{2}\left(g_{14}(k)+I g_{14}(k)\right)^{2} f_{11}^{\left(g_{14}(k)+I g_{14}(k)\right)}+\left(N_{11}(k)+\right. \\
& \left.f_{11}^{\left(g_{12}(k)+I g_{12}(k)\right)}\left(g_{12}(k)+I g_{12}(k)\right)+f_{11}^{\left(g_{14}(k)+I g_{14}(k)\right)}\left(g_{14}(k)+I g_{14}(k)\right)\right)\left(g_{11}(k)+I g_{11}(k)\right)+\frac{1}{2}\left(g_{11}(k)+\right. \\
& \left.I g_{11}(k)\right)^{2} f_{11}^{\left(g_{11}(k)+I g_{11}(k)\right)}+\left(N_{11}(k)+f_{11}^{\left(g_{12}(k)+I g_{12}(k)\right)}\left(g_{12}(k)+I g_{12}(k)\right)+f_{11}^{\left(g_{14}(k)+I g_{14}(k)\right)}\left(g_{14}(k)+I g_{14}(k)\right)+\right. \\
& \left.f_{11}^{\left(g_{11}(k)+I g_{11}(k)\right)}\left(g_{11}(k)+I g_{11}(k)\right)\right)\left(g_{13}(k)+I g_{13}(k)\right)+\frac{1}{2}\left(g_{13}(k)+I g_{13}(k)\right)^{2} f_{11}^{\left(g_{13}(k)+I g_{13}(k)\right)}- \\
& \frac{1}{2} s_{11}(k)\left(g_{11}(k)\right)^{2}-s_{11}(k) g_{11}(k)\left(\operatorname{Ig}_{11}(k)+g_{13}(k)+I g_{13}(k)\right)
\end{aligned}
$$


$D_{13}(k+1)=N_{13}(k)\left(g_{12}(k)+I g_{12}(k)\right)+\frac{1}{2}\left(g_{12}(k)+I g_{12}(k)\right)^{2}\left(f_{13}^{\left(t_{12}(k)+I g_{12}(k)\right)}\left(1-e_{13}(k)\right)+d_{13}(k)\right)+$ $\left(N_{13}(k)+\left(f_{13}^{\left(t_{12}(k)+I g_{12}(k)\right)}\left(1-e_{13}(k)\right)+d_{13}(k)\right)\left(g_{12}(k)+I g_{12}(k)\right)\right)\left(g_{14}(k)+I g_{14}(k)\right)+\frac{1}{2}\left(g_{14}(k)+\right.$ $\left.I g_{14}(k)\right)^{2}\left(f_{13}^{\left(g_{14}(k)+I g_{14}(k)\right)}\left(1-e_{13}(k)\right)+d_{13}(k)\right)+\left(N_{13}(k)+\left(f_{13}^{\left(t_{12}(k)+I g_{12}(k)\right)}\left(1-e_{13}(k)\right)+\right.\right.$ $\left.\left.d_{13}(k)\right)\left(g_{12}(k)+I g_{12}(k)\right)+\left(f_{13}^{\left(g_{14}(k)+I g_{14}(k)\right)}\left(1-e_{13}(k)\right)+d_{13}(k)\right)\left(g_{14}(k)+I g_{14}(k)\right)\right)\left(g_{11}(k)+\right.$ $\left.I g_{11}(k)\right)+\left(N_{13}(k)+\left(f_{13}^{\left(t_{12}(k)+I g_{12}(k)\right)}\left(1-e_{13}(k)\right)+d_{13}(k)\right)\left(g_{12}(k)+I_{12}(k)\right)+\left(f_{13}^{\left(g_{14}(k)+I g_{14}(k)\right)}(1-\right.\right.$ $\left.\left.\left.e_{13}(k)\right)+d_{13}(k)\right)\left(g_{14}(k)+I g_{14}(k)\right)\right)\left(g_{13}(k)+I g_{13}(k)\right)+\frac{1}{2}\left(g_{13}(k)+I g_{13}(k)\right)^{2}\left(f_{13}^{\left(g_{13}(k)+I g_{13}(k)\right)}(1-\right.$ $\left.\left.e_{13}(k)\right)+d_{13}(k)\right)-\frac{1}{2} s_{13}(k)\left(g_{13}(k)\right)^{2}-s_{13}(k) g_{13}(k) I g_{13}(k)$

Here, $f_{1 j}^{\left(g_{1 m}(k)+I g_{1 m}(k)\right)}=\frac{f_{1 j}(k)\left(g_{1 m}(k)+I g_{1 m}(k)\right)}{\left(C T_{1}\right) \max }, j=1,2,3,4, m=1,2,3,4$,

$f_{13}(k)=t_{22}(k) s_{22}(k)+t_{23}(k) s_{23}(k)+t_{24}(k) s_{24}(k)$ and $e_{13}(k)=t_{13}(k) f_{13}(k)$.

The number of vehicles at oversaturated situation and aggregate delay time of four stages at Intersection 2 are illustrated in the Figure 4:

Intersection 2

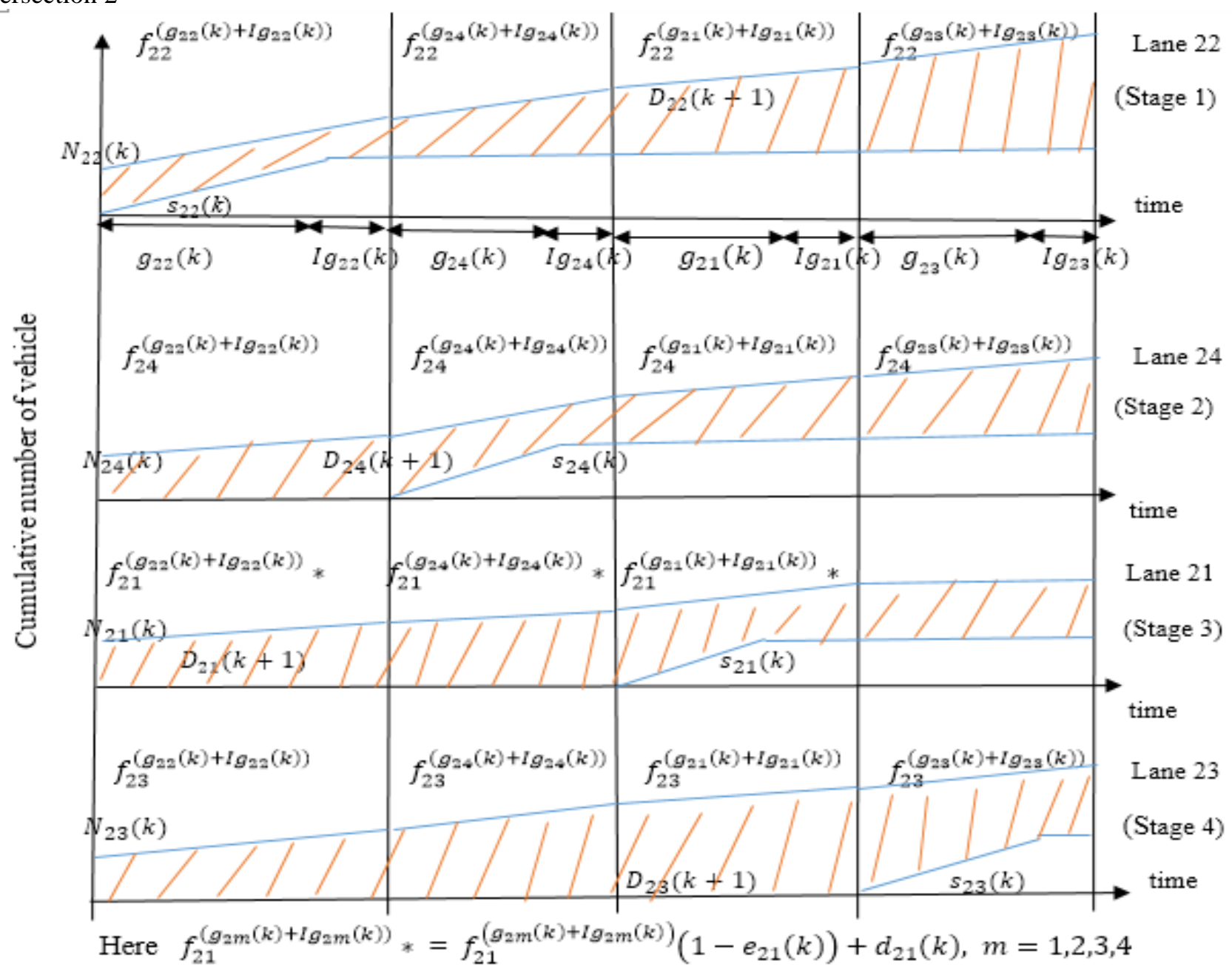

Figure 4: Number of vehicle and delay of four stages at Intersection 2 
The inequalities (Peng CHEN et al. 2013) which satisfy the oversaturation condition in each stage are given below:

$s_{22}(k) g_{22}(k)<N_{22}(k)+f_{22}^{\left(g_{22}(k)+g_{22}(k)\right)} g_{22}(k)$,

$s_{24}(k) g_{24}(k)<N_{24}(k)+f_{24}^{\left(g_{22}(k)+I g_{22}(k)\right)}\left(g_{22}(k)+I g_{22}(k)\right)+f_{24}^{\left(g_{24}(k)+I g_{24}(k)\right)} g_{24}(k)$

$s_{21}(k) g_{21}(k)<N_{21}(k)+\left(1-e_{21}(k)\right)\left(f_{21}^{\left(g_{22}(k)+I g_{22}(k)\right)}\left(g_{22}(k)+I g_{22}(k)\right)+f_{21}^{\left(g_{24}(k)+I g_{24}(k)\right)}\left(g_{24}(k)+I g_{24}(k)\right)+\right.$

$\left.f_{21}^{\left(g_{21}(k)+I g_{21}(k)\right)} g_{21}(k)\right)+d_{21}(k)\left(g_{22}(k)+I g_{22}(k)+g_{24}(k)+I g_{24}(k)+g_{21}(k)\right)$,

$s_{23}(k) g_{23}(k)<N_{23}(k)+f_{23}^{\left(g_{22}(k)+I g_{22}(k)\right)}\left(g_{22}(k)+I g_{22}(k)\right)+f_{23}^{\left(g_{24}(k)+I g_{24}(k)\right)}\left(g_{24}(k)+I g_{24}(k)\right)+$

$f_{23}^{\left(g_{21}(k)+I g_{21}(k)\right)}\left(g_{21}(k)+I g_{21}(k)\right)+f_{23}^{\left(g_{23}(k)+I g_{23}(k)\right)} g_{23}(k)$,

Here, $f_{2 j}^{\left(g_{2 m}(k)+I g_{2 m}(k)\right)}=\frac{f_{2 j}(k)\left(g_{2 m}(k)+I g_{2 m}(k)\right)}{\left(C T_{2}\right)_{\max }}, j=1,2,3,4, m=1,2,3,4$,

$f_{21}(k)=t_{11}(k) s_{11}(k)+t_{12}(k) s_{12}(k)+t_{14}(k) s_{14}(k)$ and $e_{21}(k)=t_{21}(k) f_{21}(k)$.

The aggregate delay time in each stage of the model is illustrated below:

In Stage 1 Intersection 2, the aggregate delay time for $(k+1)^{\text {th }}$ cycle (i.e. aggregate delay time at the end of $k^{\text {th }}$ cycle) is calculated by the aggregate delay time of vehicles on the Lane 22 during green signal is on and after the green signal is off which can be represented as the shaded area of Stage 1 Intersection 1 in Figure 4.

In Stage 2 Intersection 2, the aggregate delay time for $(k+1)^{\text {th }}$ cycle (aggregate delay time at the end of $k^{\text {th }}$ cycle) is calculated by the aggregate delay time of vehicles on the Lane 24 before green signal is on, during green signal is on and after the green signal is off which can be represented as the shaded area of Stage 2 Intersection 1 in Figure 4. The other two stages can also be illustrated in a similar manner.

Aggregate delay time for vehicles in each stage at the end of cycle $k$ is calculated from the area of the shaded region in the Figure 4 as given below:

$$
\begin{aligned}
& D_{22}(k+1)=N_{22}(k)\left(g_{22}(k)+I g_{22}(k)\right)+\frac{1}{2}\left(g_{22}(k)+I g_{22}(k)\right)^{2} f_{22}^{\left(t_{22}(k)+I g_{22}(k)\right)}+\left(N_{22}(k)+\right. \\
& \left.f_{22}^{\left(g_{22}(k)+I g_{22}(k)\right)}\left(g_{22}(k)+I g_{22}(k)\right)\right)\left(g_{24}(k)+I g_{24}(k)\right)+\frac{1}{2}\left(g_{24}(k)+I g_{24}(k)\right)^{2} f_{22}^{\left(g_{24}(k)+I g_{24}(k)\right)}+\left(N_{22}(k)+\right. \\
& \left.f_{22}^{\left(g_{22}(k)+I g_{22}(k)\right)}\left(g_{22}(k)+I g_{22}(k)\right)+f_{22}^{\left(g_{24}(k)+I g_{24}(k)\right)}\left(g_{24}(k)+I g_{24}(k)\right)\right)\left(g_{21}(k)+I g_{21}(k)\right)+\frac{1}{2}\left(g_{21}(k)+\right. \\
& \left.I g_{21}(k)\right)^{2} f_{22}^{\left(g_{21}(k)+I g_{21}(k)\right)}+\left(N_{22}(k)+f_{22}^{\left(g_{22}(k)+I g_{22}(k)\right)}\left(g_{22}(k)+I g_{22}(k)\right)+f_{22}^{\left(g_{24}(k)+I g_{24}(k)\right)}\left(g_{24}(k)+\right.\right. \\
& \left.\left.I g_{24}(k)\right)+f_{22}^{\left(g_{21}(k)+I g_{21}(k)\right)}\left(g_{21}(k)+I g_{21}(k)\right)\right)\left(g_{23}(k)+I g_{23}(k)\right)+\frac{1}{2}\left(g_{23}(k)+I g_{23}(k)\right)^{2} f_{22}^{\left(g_{23}(k)+I g_{23}(k)\right)}- \\
& \frac{1}{2} s_{22}(k)\left(g_{22}(k)\right)^{2}-s_{22}(k) g_{22}(k)\left(I g_{22}(k)+g_{24}(k)+I g_{24}(k)+g_{21}(k)+I g_{21}(k)+g_{23}(k)+I g_{23}(k)\right) \text {, } \\
& D_{24}(k+1)=N_{24}(k)\left(g_{22}(k)+I g_{22}(k)\right)+\frac{1}{2}\left(g_{22}(k)+I g_{22}(k)\right)^{2} f_{24}^{\left(t_{22}(k)+I g_{22}(k)\right)}+\left(N_{24}(k)+\right. \\
& \left.f_{24}^{\left(g_{22}(k)+I g_{22}(k)\right)}\left(g_{22}(k)+I g_{22}(k)\right)\right)\left(g_{24}(k)+I g_{24}(k)\right)+\frac{1}{2}\left(g_{24}(k)+I g_{24}(k)\right)^{2} f_{24}^{\left(g_{24}(k)+I g_{24}(k)\right)}+\left(N_{24}(k)+\right. \\
& \left.f_{24}^{\left(g_{22}(k)+I g_{22}(k)\right)}\left(g_{22}(k)+I g_{22}(k)\right)+f_{24}^{\left(g_{24}(k)+I g_{24}(k)\right)}\left(g_{24}(k)+I g_{24}(k)\right)\right)\left(g_{21}(k)+I g_{21}(k)\right)+\frac{1}{2}\left(g_{21}(k)+\right. \\
& \left.I g_{21}(k)\right)^{2} f_{24}^{\left(g_{21}(k)+I g_{21}(k)\right)}+\left(N_{24}(k)+f_{24}^{\left(g_{22}(k)+I g_{22}(k)\right)}\left(g_{22}(k)+I g_{22}(k)\right)+f_{24}^{\left(g_{24}(k)+I g_{24}(k)\right)}\left(g_{24}(k)+\right.\right. \\
& \left.\left.I g_{24}(k)\right)+f_{24}^{\left(g_{21}(k)+I g_{21}(k)\right)}\left(g_{21}(k)+I g_{21}(k)\right)\right)\left(g_{23}(k)+I g_{23}(k)\right)+\frac{1}{2}\left(g_{23}(k)+I g_{23}(k)\right)^{2} f_{24}^{\left(g_{23}(k)+I g_{23}(k)\right)}- \\
& \frac{1}{2} s_{24}(k)\left(g_{24}(k)\right)^{2}-s_{24}(k) g_{24}(k)\left(I_{24}(k)+g_{21}(k)+I g_{21}(k)+g_{23}(k)+I_{23}(k)\right) \text {, }
\end{aligned}
$$




$$
\begin{aligned}
& D_{21}(k+1)=N_{21}(k)\left(g_{22}(k)+I g_{22}(k)\right)+\frac{1}{2}\left(g_{22}(k)+I_{22}(k)\right)^{2}\left(f_{21}^{\left(t_{22}(k)+I g_{22}(k)\right)}\left(1-e_{21}(k)\right)+d_{21}(k)\right)+ \\
& \left(N_{21}(k)+\left(f_{21}^{\left(t_{22}(k)+I g_{22}(k)\right)}\left(1-e_{21}(k)\right)+d_{21}(k)\right)\left(g_{22}(k)+I g_{22}(k)\right)\right)\left(g_{24}(k)+I g_{24}(k)\right)+\frac{1}{2}\left(g_{24}(k)+\right. \\
& \left.I g_{24}(k)\right)^{2}\left(f_{21}^{\left(g_{24}(k)+I g_{24}(k)\right)}\left(1-e_{21}(k)\right)+d_{21}(k)\right)+\left(N_{21}(k)+\left(f_{21}^{\left(t_{22}(k)+I g_{22}(k)\right)}\left(1-e_{21}(k)\right)+\right.\right. \\
& \left.\left.d_{21}(k)\right)\left(g_{22}(k)+I g_{22}(k)\right)+\left(f_{21}^{\left(g_{24}(k)+I g_{24}(k)\right)}\left(1-e_{21}(k)\right)+d_{21}(k)\right)\left(g_{24}(k)+I g_{24}(k)\right)\right)\left(g_{21}(k)+\right. \\
& \left.I g_{21}(k)\right)+\frac{1}{2}\left(g_{21}(k)+I g_{21}(k)\right)^{2}\left(f_{21}^{\left(g_{21}(k)+I g_{21}(k)\right)}\left(1-e_{21}(k)\right)+d_{21}(k)\right)+\left(N_{21}(k)+\left(f_{21}^{\left(t_{22}(k)+I g_{22}(k)\right)}(1-\right.\right. \\
& \left.\left.e_{21}(k)\right)+d_{21}(k)\right)\left(g_{22}(k)+I g_{22}(k)\right)+\left(f_{21}^{\left(g_{24}(k)+I g_{24}(k)\right)}\left(1-e_{21}(k)\right)+d_{21}(k)\right)\left(g_{24}(k)+I g_{24}(k)\right)+ \\
& \left.\left(f_{21}^{\left(g_{21}(k)+I g_{2}(k)\right)}\left(1-e_{21}(k)\right)+d_{21}(k)\right)\left(g_{21}(k)+I g_{21}(k)\right)\right)\left(g_{23}(k)+I g_{23}(k)\right)-\frac{1}{2} s_{21}(k)\left(g_{21}(k)\right)^{2}- \\
& s_{21}(k) g_{21}(k)\left(\operatorname{Ig}_{21}(k)+g_{23}(k)+\operatorname{Ig}_{23}(k)\right) \\
& D_{23}(k+1)=N_{23}(k)\left(g_{22}(k)+I g_{22}(k)\right)+\frac{1}{2}\left(g_{22}(k)+I g_{22}(k)\right)^{2} f_{23}^{\left(t_{22}(k)+I g_{22}(k)\right)}+\left(N_{23}(k)+\right. \\
& \left.f_{23}^{\left(g_{22}(k)+I g_{22}(k)\right)}\left(g_{22}(k)+I g_{22}(k)\right)\right)\left(g_{24}(k)+I g_{24}(k)\right)+\frac{1}{2}\left(g_{24}(k)+I g_{24}(k)\right)^{2} f_{23}^{\left(g_{24}(k)+I g_{24}(k)\right)}+\left(N_{23}(k)+\right. \\
& \left.f_{23}^{\left(g_{22}(k)+I g_{22}(k)\right)}\left(g_{22}(k)+I g_{22}(k)\right)+f_{23}^{\left(g_{24}(k)+I g_{24}(k)\right)}\left(g_{24}(k)+I g_{24}(k)\right)\right)\left(g_{21}(k)+I g_{21}(k)\right)+\frac{1}{2}\left(g_{21}(k)+\right. \\
& \left.I g_{21}(k)\right)^{2} f_{23}^{\left(g_{21}(k)+I g_{21}(k)\right)}+\left(N_{23}(k)+f_{23}^{\left(g_{22}(k)+I g_{22}(k)\right)}\left(g_{22}(k)+I g_{22}(k)\right)+f_{23}^{\left(g_{24}(k)+I g_{24}(k)\right)}\left(g_{24}(k)+\right.\right. \\
& \left.\left.I g_{24}(k)\right)+f_{23}^{\left(g_{21}(k)+I g_{21}(k)\right)}\left(g_{21}(k)+I g_{21}(k)\right)\right)\left(g_{23}(k)+I g_{23}(k)\right)+\frac{1}{2}\left(g_{23}(k)+I g_{23}(k)\right)^{2} f_{23}^{\left(g_{23}(k)+I g_{23}(k)\right)}- \\
& \frac{1}{2} s_{23}(k)\left(g_{23}(k)\right)^{2}-s_{23}(k) g_{23}(k) I g_{23}(k) \text {, }
\end{aligned}
$$

Here, $f_{2 j}^{\left(g_{2 m}(k)+I g_{2 m}(k)\right)}=\frac{f_{2 j}(k)\left(g_{2 m}(k)+I g_{2 m}(k)\right)}{\left(C T_{2}\right)_{\max }}, j=1,2,3,4, m=1,2,3,4$,

$f_{21}(k)=t_{11}(k) s_{11}(k)+t_{12}(k) s_{12}(k)+t_{14}(k) s_{14}(k)$ and $e_{21}(k)=t_{21}(k) f_{21}(k)$.

The number of vehicles on eight lanes at the end of the cycle $k$ is given by the following eight equations. These equations are modified from (AhmetYazici et al. 2008). Equation (17) represents number of vehicles on Lane11 at the end of cycle $k$ (or beginning of cycle $k+1$ ) which is equivalent to the total number of vehicles waiting at the beginning of cycle $k$ (from camera readings), incoming number of vehicles into the Lane11 during green signal time, inter signal green time and red signal time and excluding outgoing number of vehicles during green signal time for the Lane 11. Similarly, the numbers of vehicles on Lane 12, Lane 14, Lane 22, Lane 23 and Lane 24 at the end of cycle $k$ (for cycle $k+1)$ are given by the equations (18), (20), (22), (23), and (24) respectively. Equation (19) represents number of vehicles on Lane 13 at the end of cycle $k$ (or beginning of cycle $k+1$ ) which is equivalent to the total of the number of vehicles waiting at the beginning of cycle $k$ (from camera readings), incoming number of vehicles into the Lane 13 during green signal time for Lane 12, Lane 13 and Lane 14 and inter signal green time, demand flow for Lane 13 during cycle time and excluding outgoing number of vehicles during green signal time for the Lane 13, exit flow for Lane 13 during cycle time. Equation (21) represents number of vehicles on Lane 21 at the end of cycle $k$ (or beginning of cycle $k+1$ ) which is equivalent to the total of the number of vehicles waiting at the beginning of cycle $k$ (from camera readings), incoming number of vehicles into the Lane 21 during green signal time for Lane 21 , Lane 22 and Lane 24 and inter signal green time, demand flow for Lane 21 during cycle time and excluding outgoing number of vehicles during green signal time for the Lane 21, exit flow for Lane 21 during cycle time. 


$$
\begin{aligned}
& N_{11}(k+1)=N_{11}(k)+f_{11}^{\left(g_{11}(k)+I g_{11}(k)\right)}\left(g_{11}(k)+I g_{11}(k)\right)+f_{11}^{\left(g_{12}(k)+I g_{12}(k)\right)}\left(g_{12}(k)+I g_{12}(k)\right)+ \\
& f_{11}^{\left(g_{13}(k)+I g_{13}(k)\right)}\left(g_{13}(k)+I g_{13}(k)\right)+f_{11}^{\left(g_{14}(k)+I g_{14}(k)\right)}\left(g_{14}(k)+I g_{14}(k)\right)-g_{11}(k) s_{11}(k), \\
& N_{12}(k+1)=N_{12}(k)+f_{12}^{\left(g_{11}(k)+I g_{11}(k)\right)}\left(g_{11}(k)+I g_{11}(k)\right)+f_{12}^{\left(g_{12}(k)+I g_{12}(k)\right)}\left(g_{12}(k)+I g_{12}(k)\right)+ \\
& f_{12}^{\left(g_{13}(k)+I g_{13}(k)\right)}\left(g_{13}(k)+I g_{13}(k)\right)+f_{12}^{\left(g_{14}(k)+I g_{14}(k)\right)}\left(g_{14}(k)+I g_{14}(k)\right)-g_{12}(k) s_{12}(k), \\
& N_{13}(k+1)=N_{13}(k)+\left(g_{11}(k)+I g_{11}(k)+g_{12}(k)+I g_{12}(k)+g_{13}(k)+I g_{13}(k)+g_{14}(k)+I g_{14}(k)\right)\left(d_{13}(k)-\right. \\
& \left.e_{13}(k)-g_{13}(k) S_{13}(k) / C_{1}\right)+f_{13}^{\left(g_{11}(k)+I g_{11}(k)\right)}\left(g_{11}(k)+I g_{11}(k)\right)+f_{13}^{\left(g_{12}(k)+I g_{12}(k)\right)}\left(g_{12}(k)+I g_{12}(k)\right)+ \\
& f_{13}^{\left(g_{13}(k)+I g_{13}(k)\right)}\left(g_{13}(k)+I g_{13}(k)\right)+f_{13}^{\left(g_{14}(k)+I g_{14}(k)\right)}\left(g_{14}(k)+I g_{14}(k)\right), \\
& N_{14}(k+1)=N_{14}(k)+f_{14}^{\left(g_{11}(k)+I g_{11}(k)\right)}\left(g_{11}(k)+I g_{11}(k)\right)+f_{14}^{\left(g_{12}(k)+I g_{12}(k)\right)}\left(g_{12}(k)+I g_{12}(k)\right)+ \\
& f_{14}^{\left(g_{13}(k)+I g_{13}(k)\right)}\left(g_{13}(k)+I g_{13}(k)\right)+f_{14}^{\left(g_{14}(k)+I g_{14}(k)\right)}\left(g_{14}(k)+I g_{14}(k)\right)-g_{14}(k) s_{14}(k), \\
& \text { Here, } f_{1 j}^{\left(g_{1 m}(k)+I g_{1 m}(k)\right)}=\frac{f_{1 j}(k)\left(g_{1 m}(k)+I g_{1 m}(k)\right)}{\left(C T_{1}\right)_{\max }}, j=1,2,3,4, m=1,2,3,4 \text {, } \\
& f_{13}(k)=t_{22}(k) s_{22}(k)+t_{23}(k) s_{23}(k)+t_{24}(k) s_{24}(k) \text { and } e_{13}(k)=t_{13}(k) f_{13}(k) \text {, } \\
& N_{21}(k+1)=N_{21}(k)+\left(g_{21}(k)+I g_{21}(k)+g_{22}(k)+I g_{22}(k)+g_{23}(k)+I g_{23}(k)+g_{24}(k)+I g_{24}(k)\right)\left(d_{21}(k)-\right. \\
& \left.e_{21}(k)-g_{21}(k) S_{21}(k) / C_{2}\right)+f_{21}^{\left(g_{21}(k)+I g_{21}(k)\right)}\left(g_{21}(k)+I g_{21}(k)\right)+f_{21}^{\left(g_{22}(k)+I g_{22}(k)\right)}\left(g_{22}(k)+I g_{22}(k)\right)+ \\
& f_{21}^{\left(g_{23}(k)+I g_{23}(k)\right)}\left(g_{23}(k)+I g_{23}(k)\right)+f_{21}^{\left(g_{24}(k)+I g_{24}(k)\right)}\left(g_{24}(k)+I g_{24}(k)\right) \text {, } \\
& N_{22}(k+1)=N_{22}(k)+f_{22}^{\left(g_{21}(k)+I g_{21}(k)\right)}\left(g_{21}(k)+I g_{21}(k)\right)+f_{22}^{\left(g_{22}(k)+I g_{22}(k)\right)}\left(g_{22}(k)+I g_{22}(k)\right)+ \\
& f_{22}^{\left(g_{23}(k)+I g_{23}(k)\right)}\left(g_{23}(k)+I g_{23}(k)\right)+f_{22}^{\left(g_{24}(k)+I g_{24}(k)\right)}\left(g_{24}(k)+I g_{24}(k)\right)-g_{22}(k) s_{22}(k), \\
& N_{23}(k+1)=N_{23}(k)+f_{23}^{\left(g_{21}(k)+I g_{21}(k)\right)}\left(g_{21}(k)+I g_{21}(k)\right)+f_{23}^{\left(g_{22}(k)+I g_{22}(k)\right)}\left(g_{22}(k)+I g_{22}(k)\right)+ \\
& f_{23}^{\left(g_{23}(k)+I g_{23}(k)\right)}\left(g_{23}(k)+I g_{23}(k)\right)+f_{23}^{\left(g_{24}(k)+I g_{24}(k)\right)}\left(g_{24}(k)+I g_{24}(k)\right)-g_{23}(k) s_{23}(k), \\
& N_{24}(k+1)=N_{24}(k)+f_{21}^{\left(g_{21}(k)+I g_{21}(k)\right)}\left(g_{21}(k)+I g_{21}(k)\right)+f_{21}^{\left(g_{22}(k)+I g_{22}(k)\right)}\left(g_{22}(k)+I g_{22}(k)\right)+ \\
& f_{21}^{\left(g_{23}(k)+I g_{23}(k)\right)}\left(g_{23}(k)+I g_{23}(k)\right)+f_{21}^{\left(g_{24}(k)+I g_{24}(k)\right)}\left(g_{24}(k)+I g_{24}(k)\right)-g_{24}(k) s_{24}(k) \\
& \text { Here, } f_{2 j}^{\left(g_{2 m}(k)+I g_{2 m}(k)\right)}=\frac{f_{2 j}(k)\left(g_{2 m}(k)+I g_{2 m}(k)\right)}{\left(C T_{2}\right) \max }, j=1,2,3,4, m=1,2,3,4 \text {, } \\
& f_{21}(k)=t_{11}(k) s_{11}(k)+t_{12}(k) s_{12}(k)+t_{14}(k) s_{14}(k) \text { and } e_{21}(k)=t_{21}(k) f_{21}(k) \text {. }
\end{aligned}
$$

Lower and upper bounds of green signal time are given by,$$
\left(g_{i j}(k)\right)_{\min } \leq g_{i j}(k) \leq\left(g_{i j}(k)\right)_{\max }, i=1,2, j=1,2,3,4 \text {, }
$$

Cycle time of cycle $\mathrm{k}$ is given by the total green signal time and total inter green signal time:

$\sum_{j=1}^{4}\left(g_{i j}(k)+I g_{i j}(k)\right)=C_{i}, i=1,2$.

Lower and upper bounds of cycle time are given by,

$$
\left(C T_{i}\right)_{\min } \leq C_{i}(k) \leq\left(C T_{i}\right)_{\max }, i=1,2 \text {. }
$$

The incoming number of vehicles into a lane during a cycle time is less than or equal to the outgoing number of vehicles from that lane during green signal time is considered as a condition. The following eight inequalities represent those conditions during cycle $k$ for the Lane 11, Lane 12, Lane 13, Lane 14, Lane 21, Lane 22, Lane 23 and Lane 24 respectively:

$$
\begin{aligned}
& f_{11}^{\left(g_{11}(k)+I g_{11}(k)\right)}\left(g_{11}(k)+I g_{11}(k)\right)+f_{11}^{\left(g_{12}(k)+I g_{12}(k)\right)}\left(g_{12}(k)+I g_{12}(k)\right)+f_{11}^{\left(g_{13}(k)+I g_{13}(k)\right)}\left(g_{13}(k)+I g_{13}(k)\right)+ \\
& f_{11}^{\left(g_{14}(k)+I g_{14}(k)\right)}\left(g_{14}(k)+I g_{14}(k) \leq s_{11}(k) g_{11}(k),\right. \\
& f_{12}^{\left(g_{11}(k)+I g_{11}(k)\right)}\left(g_{11}(k)+I g_{11}(k)\right)+f_{12}^{\left(g_{12}(k)+I g_{12}(k)\right)}\left(g_{12}(k)+I g_{12}(k)\right)+f_{12}^{\left(g_{13}(k)+I g_{13}(k)\right)}\left(g_{13}(k)+I g_{13}(k)\right)+ \\
& f_{12}^{\left(g_{14}(k)+I g_{14}(k)\right)}\left(g_{14}(k)+I g_{14}(k) \leq s_{12}(k) g_{12}(k),\right.
\end{aligned}
$$




$$
\begin{aligned}
& \left(1-e_{13}(k)\right)\left(f_{13}^{\left(g_{12}(k)+I g_{12}(k)\right)}\left(g_{12}(k)+I g_{12}(k)\right)+f_{13}^{\left(g_{13}(k)+I g_{13}(k)\right)}\left(g_{13}(k)+I g_{13}(k)\right)+\right. \\
& \left.f_{13}^{\left(g_{14}(k)+I g_{14}(k)\right)}\left(g_{14}(k)+I g_{14}(k)\right)\right)+d_{13}(k)\left(g_{12}(k)+I g_{12}(k)+g_{13}(k)+I g_{13}(k)+g_{14}(k)+I g_{14}(k)\right) \leq \\
& s_{13}(k) g_{13}(k) \text {, } \\
& f_{14}^{\left(g_{11}(k)+I g_{11}(k)\right)}\left(g_{11}(k)+I g_{11}(k)\right)+f_{14}^{\left(g_{12}(k)+I g_{12}(k)\right)}\left(g_{12}(k)+I g_{12}(k)\right)+f_{14}^{\left(g_{13}(k)+I g_{13}(k)\right)}\left(g_{13}(k)+I g_{13}(k)\right)+ \\
& f_{14}^{\left(g_{14}(k)+I g_{14}(k)\right)}\left(g_{14}(k)+I g_{14}(k) \leq s_{14}(k) g_{14}(k)\right. \text {, } \\
& \text { Here, } f_{1 j}^{\left(g_{1 m}(k)+I g_{1 m}(k)\right)}=\frac{f_{1 j}(k)\left(g_{1 m}(k)+I g_{1 m}(k)\right)}{\left(C T_{1}\right) \max }, j=1,2,3,4, m=1,2,3,4 \text {, } \\
& f_{13}(k)=t_{22}(k) s_{22}(k)+t_{23}(k) s_{23}(k)+t_{24}(k) s_{24}(k) \text { and } e_{13}(k)=t_{13}(k) f_{13}(k) \text {. } \\
& \left(1-e_{21}(k)\right)\left(f_{21}^{\left(g_{21}(k)+I g_{21}(k)\right)}\left(g_{21}(k)+I g_{21}(k)\right)+f_{21}^{\left(g_{22}(k)+I g_{22}(k)\right)}\left(g_{22}(k)+I g_{22}(k)\right)+\right. \\
& \left.f_{21}^{\left(g_{24}(k)+I g_{24}(k)\right)}\left(g_{24}(k)+I g_{24}(k)\right)\right)+d_{21}(k)\left(g_{21}(k)+I g_{21}(k)+g_{22}(k)+I g_{22}(k)+g_{24}(k)+I g_{24}(k)\right) \leq \\
& s_{21}(k) g_{21}(k) \text {, } \\
& f_{22}^{\left(g_{21}(k)+I g_{21}(k)\right)}\left(g_{21}(k)+I g_{21}(k)\right)+f_{22}^{\left(g_{22}(k)+I g_{22}(k)\right)}\left(g_{22}(k)+I g_{22}(k)\right)+f_{22}^{\left(g_{23}(k)+I g_{23}(k)\right)}\left(g_{23}(k)+I g_{23}(k)\right)+ \\
& f_{22}^{\left(g_{24}(k)+I g_{24}(k)\right)}\left(g_{24}(k)+I g_{24}(k) \leq s_{22}(k) g_{22}(k)\right. \text {, } \\
& f_{23}^{\left(g_{21}(k)+I g_{21}(k)\right)}\left(g_{21}(k)+I g_{21}(k)\right)+f_{23}^{\left(g_{22}(k)+I g_{22}(k)\right)}\left(g_{22}(k)+I g_{22}(k)\right)+f_{23}^{\left(g_{23}(k)+I g_{23}(k)\right)}\left(g_{23}(k)+I g_{23}(k)\right)+ \\
& f_{23}^{\left(g_{24}(k)+g_{24}(k)\right)}\left(g_{24}(k)+I g_{24}(k) \leq s_{23}(k) g_{23}(k)\right. \text {, } \\
& f_{24}^{\left(g_{21}(k)+I g_{21}(k)\right)}\left(g_{21}(k)+I g_{21}(k)\right)+f_{24}^{\left(g_{22}(k)+I g_{22}(k)\right)}\left(g_{22}(k)+I g_{22}(k)\right)+f_{24}^{\left(g_{23}(k)+I g_{23}(k)\right)}\left(g_{23}(k)+I g_{23}(k)\right)+ \\
& f_{24}^{\left(g_{24}(k)+\operatorname{Ig}_{24}(k)\right)}\left(g_{24}(k)+\operatorname{Ig}_{24}(k) \leq s_{24}(k) g_{24}(k)\right. \text {, } \\
& \text { Here, } f_{2 j}^{\left(g_{2 m}(k)+I g_{2 m}(k)\right)}=\frac{f_{2 j}(k)\left(g_{2 m}(k)+I g_{2 m}(k)\right)}{\left(C T_{2}\right) \max }, j=1,2,3,4, m=1,2,3,4 \text {, } \\
& f_{21}(k)=t_{11}(k) s_{11}(k)+t_{12}(k) s_{12}(k)+t_{14}(k) s_{14}(k) \text { and } e_{21}(k)=t_{21}(k) f_{21}(k) \text {. }
\end{aligned}
$$

A special oversaturation condition is defined as $\alpha$ times the number of outgoing vehicles during green signal time on a lane at a given cycle is less than the number of vehicles on that lane at the end of that cycle. This condition satisfies the oversaturation conditions given in equations (1), (2), (3), (4), (9), (10), (11) and (12) along with above conditions given in equations (28), (29), (30), (31), (32), (33), (34) and (35). The following inequality represents the special oversaturation condition:

$\alpha s_{i j}(k) g_{i j}(k) \leq N_{i j}(k+1), i=1,2, j=1,2,3,4$ and $\alpha$ is a positive number. Upper bound of $\alpha$ depends on the other variable values of the problem.

When the value of $\alpha$ decrease the number of waiting vehicles at the end of the final cycle will decrease and number of cycles to converge to final cycle will increase. In this model two better cases are considered.

Case I $(\alpha=2)$

The following eight inequalities represent the special oversaturation conditions during cycle $k$ for the Lane 11, Lane 12, Lane 13, Lane 14, Lane 21, Lane 22, Lane 23 and Lane 24 respectively:

$2 s_{i j}(k) g_{i j}(k) \leq N_{i j}(k+1), i=1,2, j=1,2,3,4$.

Case II $(\alpha=1)$

The following eight inequalities represent the special oversaturation conditions during cycle $k$ for the Lane 11, Lane 12, Lane 13, Lane 14, Lane 21, Lane 22, Lane 23 and Lane 24 respectively: $s_{i j}(k) g_{i j}(k) \leq N_{i j}(k+1), i=1,2, j=1,2,3,4$. 


\section{Flow chart of the model and the method of solution}

Obtain the number of vehicles on eachlane from camera

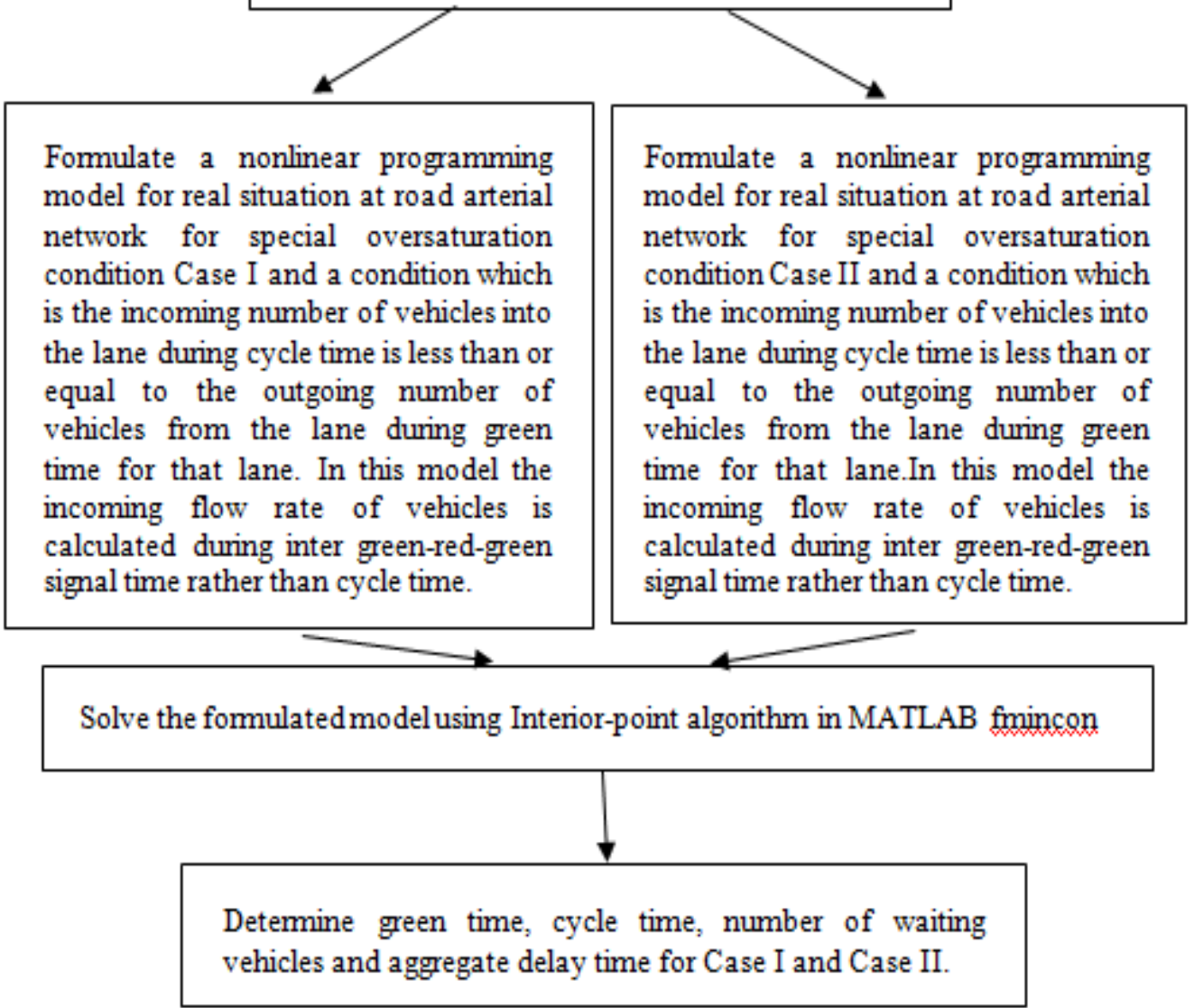

Figure 5: Flow chart of the model and the solution.

\section{Nonlinear programming problem model}

Objectives of the nonlinear programming problem model under Case I and nonlinear programming problem model under Case II are to minimize the total number of vehicles waiting at the intersections and aggregate delay times subject to the Case I oversaturation and Case II oversaturation conditions respectively, and the delay, additional conditions and some constraints related to the traffic signal control problem, which are described above, are combined into the models formulation and is illustrated in sections 4.1 and 4.2 respectively to calculate the duration of the green signal time at the beginning of the cycle $k$ :In both models:

- The cycle time is equal to the sum of the total green signal time and total inter green time. Each green time value has a minimum value $\left(\left(g_{i j}(k)\right)_{\min }\right)$ and a maximum value $\left(\left(g_{i j}(k)\right)_{\max }\right)$ which are fixed for a cycle.

- In order to maintain the feasibility, the sum of the total minimum green signal time values and inter green signal time values is assumed to be greater than or equal to $\left(C T_{i}\right)_{\text {min }}, i=1,2$ and also, the sum of the total maximum green signal time values and inter green signal time values is assumed to be less than or equal to $\left(C T_{i}\right)_{\max } . i=1,2$.

- The objective function consists of waiting parameters $W_{i j}, i=1,2, j=1,2,3,4$ assigned to each lane at each intersection. The default value of $W_{i j}, i=1,2, j=1,2,3,4$ is assigned to 1 . The objective function can be optimized by selecting different waiting parameters $W_{i j}$ according to different criteria: lane priority, emergency vehicle passing etc.

- To optimize green time for each signal we apply interior point algorithm implemented in the MATLAB optimization toolbox for the above nonlinear programming problem. 


\subsection{Nonlinear programming problem model for Case I}

Minimize $Z=\sum_{j=1}^{4} \sum_{i=1}^{2} W_{i j} N_{i j}(k+1)$

Subject to

Equations (17), (18), (19), (20), (21), (22), (23), (24) and Inequalities (25), (26), (27), (28), (29), (30), (31), (32), (33), (34), (35), (36), $D_{i j}(k+1) \geq 0, \quad i=1,2, \quad j=1,2,3,4$.

\subsection{Nonlinear programming problem model for Case II}

Minimize $Z=\sum_{j=1}^{4} \sum_{i=1}^{2} W_{i j} N_{i j}(k+1)$

Subject to

Equations (17), (18), (19), (20), (21), (22), (23), (24) and Inequalities (25), (26), (27), (28), (29), (30), (31), (32), (33), (34), (35), (37), $D_{i j}(k+1) \geq 0, \quad i=1,2, \quad j=1,2,3,4$.

\section{Results and discussion}

One arterial network is considered to discuss these proposed models. Hypothetical data set is applied to find optimum solution of the nonlinear programming problem model for each Case I and Case II.

\subsection{Results and discussion for hypothetical data set of arterial network for Case I and Case II}

Hypothetical data set:

Distance between Intersection 1 and Intersection 2 is $700 \mathrm{~m}$. Average length of a small vehicle is approximately $5 \mathrm{~m}$. So the number of vehicles on Lane 13 and Lane 21 is less than 140.

Distance between upstream camera and downstream camera installed on any lane is $400 \mathrm{~m}$. If we assume that the average length of a small vehicle is approximately $5 \mathrm{~m}$, then the maximum number of vehicles on a lane within $400 \mathrm{~m}$ is 80 .

Incoming flow rates of vehicles for the lanes are fixed over the cycles is given by

$f_{11}=0.19$ vehicles $/ \mathrm{sec}_{\text {. }}, f_{12}=0.15$ vehicles $/ \mathrm{sec}_{\text {, }} f_{14}=0.15$ vehicles $/ \mathrm{sec} ., f_{22}=0.16$ vehicles $/$ sec. $f_{23}=0.19$ vehicles $/ \mathrm{sec}$.,$f_{24}=0.16$ vehicles $/$ sec..

Outgoing flow rates of vehicles for the lanes are fixed over the cycles is given by $s_{i j}=0.45$ vehicles $/$ sec. $, ~ i=1,2, j=1,2,3,4$.

Inter green signal time is given by $I g_{i j}=4 \mathrm{sec}_{.}, i=1,2, j=1,2,3,4$.

Vehicle turning rates are fixed over the cycles:

$t_{11}=0.55, t_{12}=0.12, t_{14}=0.05, t_{22}=0.05, t_{23}=0.55, t_{24}=0.12$.

Saturation flow rates are fixed over the cycles: $S_{13}(k)=0.45, S_{21}(k)=0.45$.

Vehicle exit flow is fixed over the cycles: $e_{13}=0.6, e_{21}=0.6$.

Vehicle demand flow is fixed over the cycles: $d_{13}=0, d_{21}=0$.

The upper bound of cycle time is fixed to $120 \mathrm{sec}$. The lower bound of cycle time is attained according to the model. Similarly, the upper bound and lower bound of green signal times are attained according to the model.

\subsubsection{Results and discussion for hypothetical data set of arterial network for Case I}

Simulation results are given in the following Table 1 :

From camera readings: $N_{11}(1)=68, N_{12}(1)=46, N_{13}(1)=45, N_{14}(1)=51$,

$$
N_{21}(1)=47, N_{22}(1)=57, N_{23}(1)=61, \quad N_{24}(1)=59 \text {. }
$$

Phase sequence order (signal order):

Intersection 1: Lane 12 signal, Lane 14 signal, Lane 11 signal, Lane 13 signal.

Intersection 2: Lane 22 signal, Lane 24 signal, Lane 21 signal, Lane 23 signal. 
Table 1: Cycles and the optimum feasible results for arterial network for Case I

\begin{tabular}{|c|c|c|c|c|c|c|}
\hline $\begin{array}{l}\text { C } \\
\text { yc } \\
\text { le }\end{array}$ & $\begin{array}{l}\text { Number of vehicles on } \\
\text { lanes at each } \\
\text { intersection and total } \\
\text { of that at the } \\
\text { beginning of the cycle, } \\
j=1,2,3,4 \\
\qquad N_{1 j}(k)\left[N T_{1}(k)\right] \\
\quad N_{2 j}(k)\left[N T_{2}(k)\right]\end{array}$ & $\begin{array}{l}\text { Green } \\
\text { time } \\
\text { each } \\
\text { intersection } \\
t_{1 j}(k) \\
t_{2 j}(k) \\
j=1,2,\end{array}$ & & $\begin{array}{l}\text { Cycle } \\
\text { time } \\
\text { (sec.) } \\
C_{1}(k) \\
C_{2}(k)\end{array}$ & $\begin{array}{l}\text { Aggregate delay time and total of } \\
\text { that at each intersection at the } \\
\text { end of the cycle (sec.) } \\
\qquad D_{1 j}(k+1)\left[D T_{1}(k+1)\right] \\
D_{2 j}(k+1)\left[D T_{2}(k+1)\right] \\
\quad j=1,2,3,4\end{array}$ & $\begin{array}{l}\text { Number of } \\
\text { vehicles on } \\
\text { lanes at each } \\
\text { intersection at } \\
\text { the end of the } \\
\text { cycle. } \\
N_{1 j}(k+1) \\
N_{2 j}(k+1) \\
j=1,2,3,4\end{array}$ \\
\hline \multirow[t]{2}{*}{01} & $\begin{array}{lllll}68 & 46 & 45 & 51 & {[210]}\end{array}$ & $27 \quad 24 \quad 27$ & & 118 & $\begin{array}{lllll}7753 & 4534 & 5266 & 5426 & {[22979]}\end{array}$ & $61 \quad 40 \quad 35 \quad 45$ \\
\hline & $\begin{array}{llll}47 & 57 & 61 & 59\end{array}$ & $24 \quad 27$ & 24 & 118 & $\begin{array}{lllll}5157 & 5849 & 7303 & 6331 & {[24640]}\end{array}$ & $\begin{array}{llll}37 & 51 & 54 & 53\end{array}$ \\
\hline \multirow[t]{2}{*}{02} & $\begin{array}{llll}61 & 40 & 35 & 45\end{array}$ & 24 & 24 & 118 & $\begin{array}{lllll}6927 & 3826 & 4086 & 4718 & {[19557]}\end{array}$ & $54 \quad 342539$ \\
\hline & $\begin{array}{llll}37 & 51 & 54 & 53\end{array}$ & $24 \quad 27$ & 24 & 118 & $\begin{array}{lllll}3977 & 5141 & 6477 & 5623 & {[212}\end{array}$ & $27 \quad 45 \quad 47 \quad 47$ \\
\hline \multirow[t]{2}{*}{03} & $\begin{array}{llll}54 & 34 & 25 & 39\end{array}$ & $28 \quad 20$ & 32 & 117 & $\begin{array}{lllll}6302 & 2955 & 3012 & 3844 & {[16113]}\end{array}$ & $\begin{array}{llll}50 & 26 & 18 & 29\end{array}$ \\
\hline & 2745 & 28 & 32 & 117 & $3025 \quad 4261 \quad 5721 \quad 4727[17734]$ & $20 \quad 37 \quad 4437$ \\
\hline \multirow[t]{2}{*}{04} & $\begin{array}{llll}50 & 26 & 18 & 29\end{array}$ & $21 \quad 14$ & 23 & 89 & $\begin{array}{lllll}4405 & 1694 & 1627 & 2159 & {[9885]}\end{array}$ & $\begin{array}{llll}46 & 19 & 13 & 21\end{array}$ \\
\hline & $\begin{array}{llll}37 & 44 & 37 & {[138]}\end{array}$ & 21 & 23 & 89 & $\begin{array}{lllll}1683 & 2681 & 4001 & 2880 & {[11245]}\end{array}$ & $15 \quad 30 \quad 41 \quad 29$ \\
\hline \multirow[t]{2}{*}{05} & $\begin{array}{lllll}46 & 19 & 13 & 21 & {[99]}\end{array}$ & 15 & 16 & 64 & $\begin{array}{lllll}3080 & 937 & 885 & 1186 & {[6088]}\end{array}$ & $\begin{array}{llll}43 & 14 & 09 & 15\end{array}$ \\
\hline & $\begin{array}{llll}15 & 30 & 41 & 29\end{array}$ & 15 & 16 & 64 & $\begin{array}{lllll}949 & 1689 & 2815 & 1754\end{array}$ & $\begin{array}{llll}11 & 25 & 38 & 23\end{array}$ \\
\hline \multirow[t]{2}{*}{06} & $\begin{array}{llll}43 & 14 & 09 & 15\end{array}$ & 1106 & 11 & 52 & $\begin{array}{llll}2202 & 522 & 468 & 648\end{array}$ & $\begin{array}{llll}40 & 10 & 07 & 11\end{array}$ \\
\hline & $\begin{array}{llll}11 & 25 & 38 & 23\end{array}$ & 1106 & 11 & 52 & $\begin{array}{llll}528 & 1096 & 1988 & 1096\end{array}$ & $\begin{array}{llll}08 & 21 & 36 & 19\end{array}$ \\
\hline \multirow[t]{2}{*}{07} & $\begin{array}{llll}40 & 10 & 07 & 11\end{array}$ & $07 \quad 05$ & 08 & 42 & $\begin{array}{llll}1652 & 311 & 288 & 377\end{array}$ & $\begin{array}{llll}38 & 07 & 05 & 08\end{array}$ \\
\hline & $\begin{array}{llll}08 & 21 & 36 & 19\end{array}$ & $07 \quad 05$ & 08 & 42 & $\begin{array}{lll}774 & 1513 \quad 738\end{array}$ & $\begin{array}{llll}05 & 18 & 34 & 16\end{array}$ \\
\hline \multirow[t]{2}{*}{08} & $\begin{array}{llll}38 & 07 & 05 & 08\end{array}$ & $\begin{array}{ll}05 & 03\end{array}$ & 06 & 33 & $\begin{array}{lll}168 & 162 & 213\end{array}$ & $\begin{array}{llll}37 & 05 & 04 & 06\end{array}$ \\
\hline & $\begin{array}{llll}05 & 18 & 34 & 16\end{array}$ & 05 & 06 & 33 & 5321122 & $\begin{array}{llll}04 & 16 & 33 & 14\end{array}$ \\
\hline \multirow[t]{2}{*}{09} & $\begin{array}{llll}37 & 05 & 04 & 06\end{array}$ & 03 & 04 & 27 & 104106 & $\begin{array}{llll}36 & 04 & 03 & 04\end{array}$ \\
\hline & $\begin{array}{llll}04 & 16 & 33 & 14\end{array}$ & $03 \quad 02$ & 04 & 27 & 401 & $\begin{array}{llll}03 & 15 & 32 & 12\end{array}$ \\
\hline \multirow[t]{2}{*}{10} & $\begin{array}{llll}36 & 04 & 03 & 04\end{array}$ & 02 & 03 & 26 & 930 & $\begin{array}{llll}35 & 03 & 02 & 03\end{array}$ \\
\hline & $\begin{array}{llll}03 & 15 & 32 & 12\end{array}$ & 02 & 03 & 26 & {$[1573]$} & $\begin{array}{llll}02 & 14 & 31 & 11\end{array}$ \\
\hline \multirow[t]{2}{*}{11} & \begin{tabular}{|lllll}
35 & 03 & 02 & 03
\end{tabular} & 02 & 02 & 22 & [ 914] & $\begin{array}{llll}35 & 02 & 02 & 02\end{array}$ \\
\hline & $\begin{array}{llll}02 & 14 & 31 & 11\end{array}$ & 01 & 02 & 22 & {$[1262]$} & $\begin{array}{llll}02 & 13 & 31 & 10\end{array}$ \\
\hline \multirow[t]{2}{*}{12} & $\begin{array}{llll}35 & 02 & 02 & 02\end{array}$ & $\begin{array}{ll}01 & 01\end{array}$ & 01 & 20 & [ 803] & $\begin{array}{llll}35 & 02 & 02 & 02\end{array}$ \\
\hline & $\begin{array}{llll}02 & 13 & 31 & 10\end{array}$ & 01 & 01 & 20 & {$[1120]$} & $\begin{array}{llll}02 & 13 & 31 & 10\end{array}$ \\
\hline \multirow[t]{2}{*}{13} & $\begin{array}{llll}35 & 02 & 02 & 02\end{array}$ & 01 & 01 & 20 & [ 803] & $\begin{array}{llll}35 & 02 & 02 & 02\end{array}$ \\
\hline & $\begin{array}{llll}02 & 13 & 31 & 10\end{array}$ & 0101 & 01 & 20 & {$[1120]$} & $\begin{array}{llll}02 & 13 & 31 & 10\end{array}$ \\
\hline
\end{tabular}

In the Table 1 given above, the results of cycle 12 and cycle 13 are the same. If this process continues into more cycles, the results will be the same as the results of cycle 12. Because of oversaturation situation some vehicles are still waiting on each lane in the last cycle (cycle 12). 


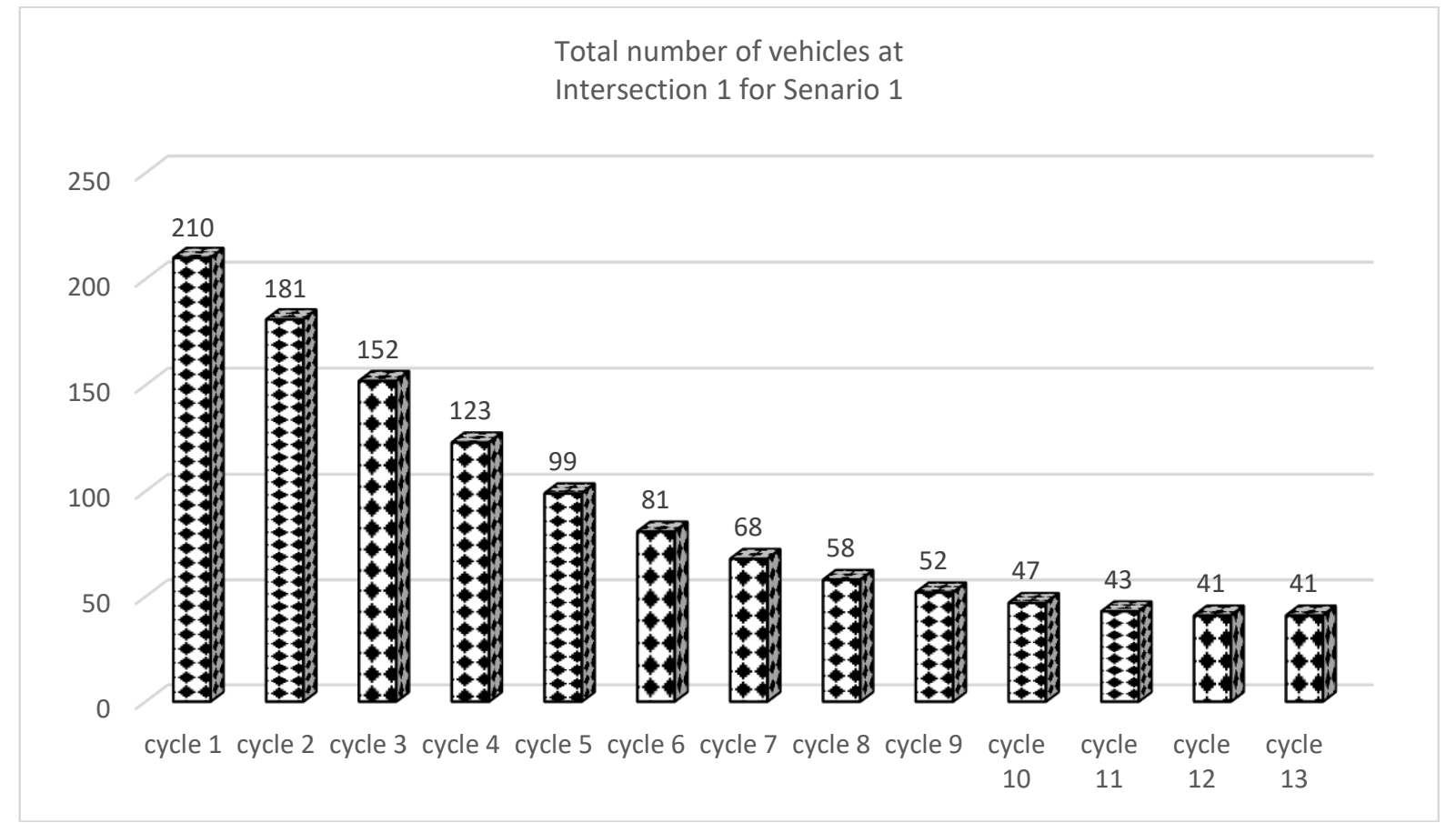

Figure 6: Total number of vehicles in each cycle at Intersection 1 of arterial network for Case I.

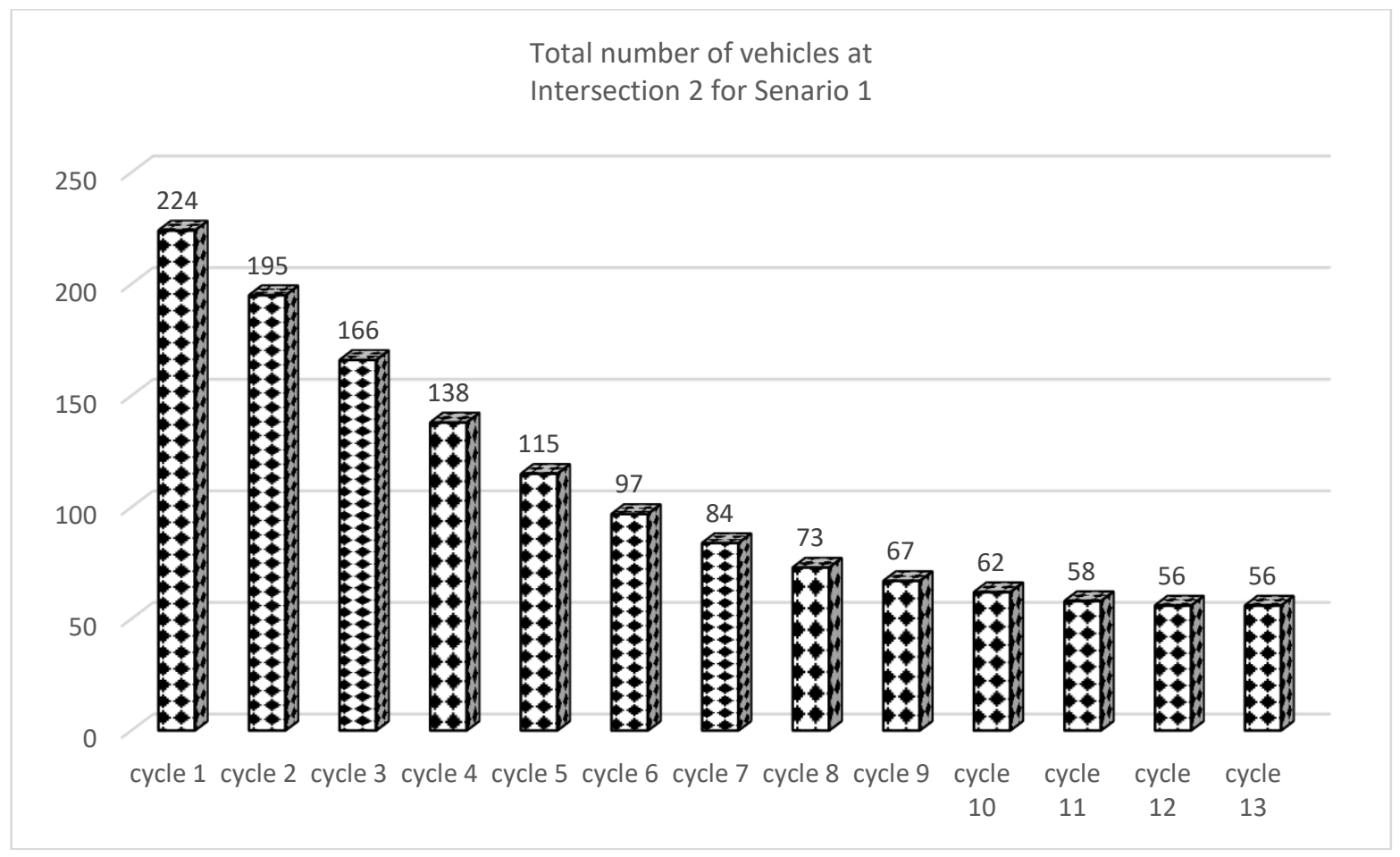

Figure 7: Total number of vehicles in each cycle at Intersection 2 of arterial network for Case I. 


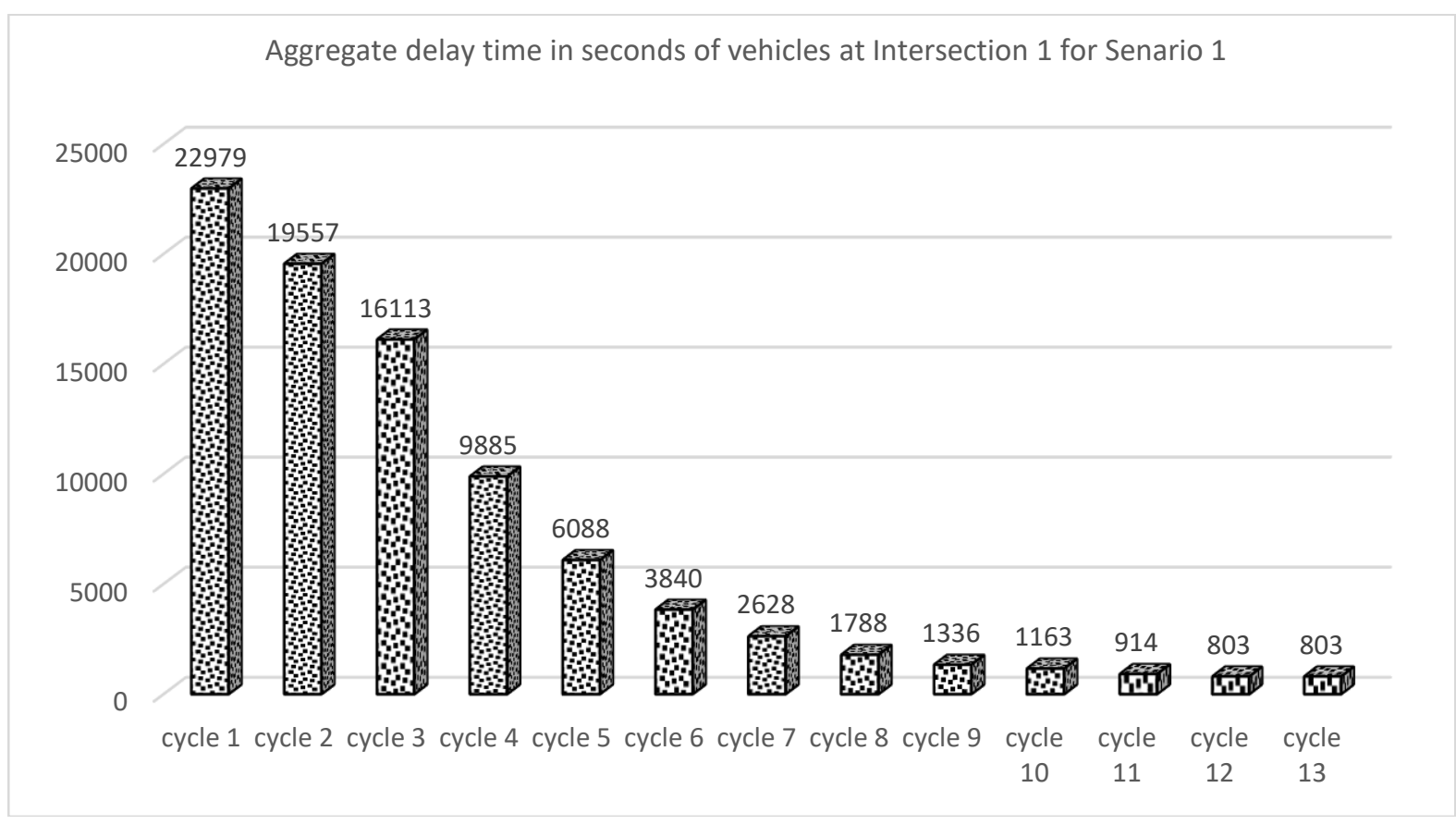

Figure 8: Aggregate delay time in each cycle at Intersection 1 of arterial network for Case I.

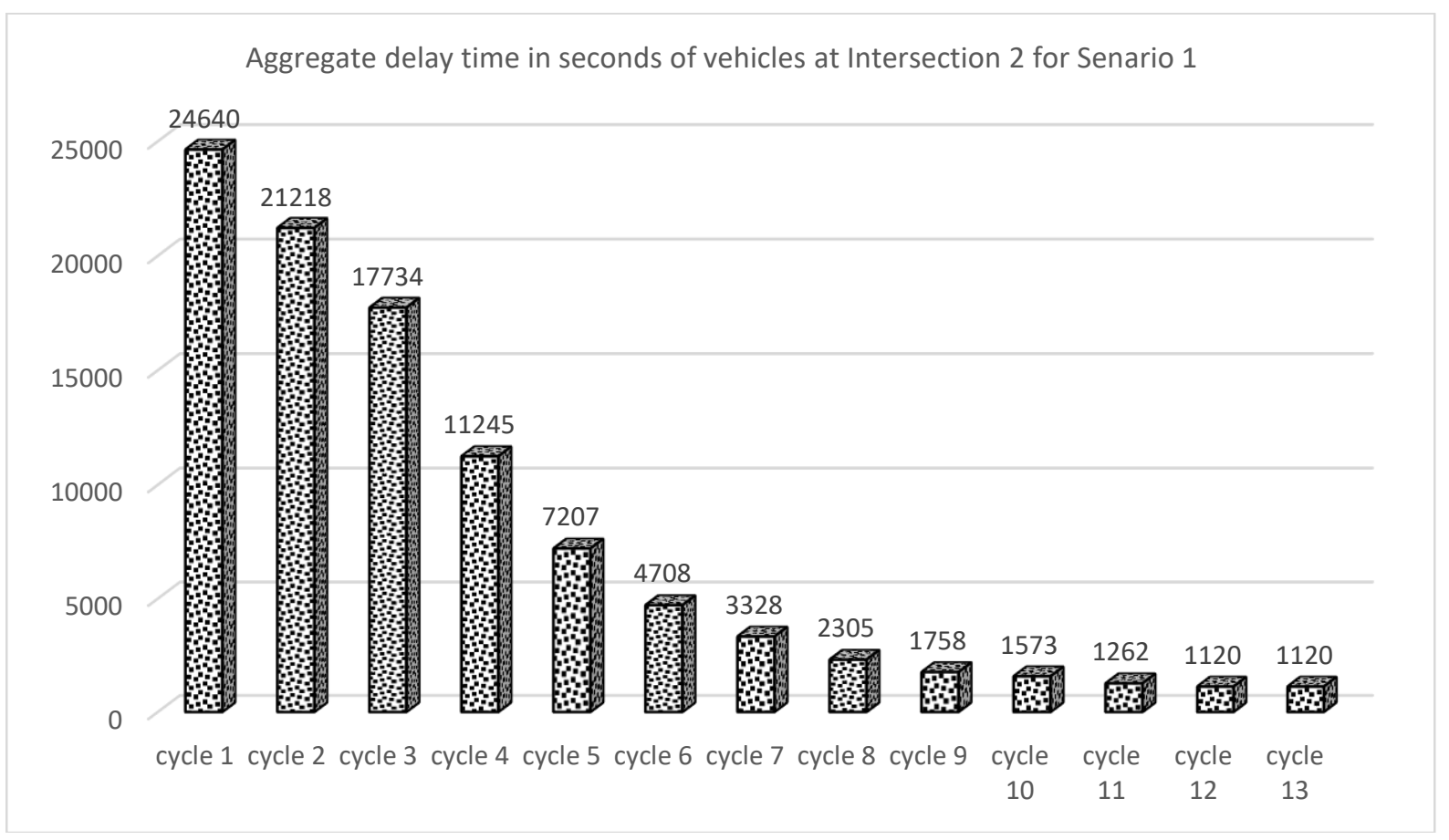

Figure 9: Aggregate delay time in each cycle at Intersection 2 of arterial network for Case I.

\subsubsection{Results and discussion for hypothetical data set of arterial network for Case II}

Simulation results are given in the following Table 2:

From camera readings: $N_{11}(1)=70, N_{12}(1)=45, N_{13}(1)=48, N_{14}(1)=41$,

$$
N_{21}(1)=39, N_{22}(1)=53, N_{23}(1)=74, \quad N_{24}(1)=58 \text {. }
$$

Phase sequence order (signal order):

Intersection 1: Lane 11 signal, Lane 13 signal, Lane 12 signal, Lane 14 signal.

Intersection 2: Lane 23 signal, Lane 24 signal, Lane 22 signal, Lane 21 signal. 
Table 2: Cycles and the optimum feasible results for arterial network for Case II

\begin{tabular}{|c|c|c|c|c|c|}
\hline $\begin{array}{l}\text { Cy } \\
\text { cle } \\
k\end{array}$ & $\begin{array}{l}\text { Number of vehicles } \\
\text { on lanes at each } \\
\text { intersection and total } \\
\text { of that at the } \\
\text { beginning of the } \\
\text { cycle, } j=1,2,3,4 \\
N_{1 j}(k)\left[N T_{1}(k)\right] \\
N_{2 j}(k)\left[N T_{2}(k)\right]\end{array}$ & $\begin{array}{l}\text { Green signal } \\
\text { time (sec.) at } \\
\text { each } \\
\text { intersection } \\
\qquad \begin{array}{l}t_{1 j}(k) \\
\quad t_{2 j}(k) \\
j=1,2,3,4\end{array}\end{array}$ & $\begin{array}{l}\text { Cycle } \\
\text { time } \\
(\text { sec.) } \\
C_{1}(k) \\
C_{2}(k)\end{array}$ & $\begin{array}{l}\text { Aggregate delay time and total of } \\
\text { that at each intersection at the end } \\
\text { of the cycle (sec.) } \\
\qquad \begin{array}{l}D_{1 j}(k+1)\left[D T_{1}(k+1)\right] \\
D_{2 j}(k+1)\left[D T_{2}(k+1)\right] \\
\quad j=1,2,3,4\end{array}\end{array}$ & $\begin{array}{l}\text { Number of } \\
\text { vehicles on } \\
\text { lanes at each } \\
\text { intersection at } \\
\text { the end of the } \\
\text { cycle. } \\
N_{1 j}(k+1) \\
N_{2 j}(k+1) \\
j=1,2,3,4 \\
\end{array}$ \\
\hline 01 & $\begin{array}{lllll}70 & 45 & 48 & 41 & {[204]} \\
39 & 53 & 74 & 58 & {[224]} \\
\end{array}$ & $\begin{array}{llll}27 & 24 & 27 & 24 \\
27 & 24 & 27 & 24 \\
\end{array}$ & $\begin{array}{l}118 \\
118\end{array}$ & $\begin{array}{lllll}7989 & 4416 & 5620 & 4246 & {[22271]} \\
4213 & 5377 & 8837 & 6129 & {[24556]} \\
\end{array}$ & $\begin{array}{llll}63 & 39 & 38 & 35 \\
29 & 47 & 67 & 52 \\
\end{array}$ \\
\hline 02 & $\begin{array}{lllll}63 & 39 & 38 & 35 & {[175]} \\
29 & 47 & 67 & 52 & {[195]} \\
\end{array}$ & $\begin{array}{llll}27 & 24 & 27 & 24 \\
27 & 24 & 27 & 24 \\
\end{array}$ & $\begin{array}{l}118 \\
118\end{array}$ & $\begin{array}{llllll}7163 & 3708 & 4440 & 3538 & {[18849]} \\
3033 & 4669 & 8011 & 5421 & {[21134]} \\
\end{array}$ & $\begin{array}{llll}56 & 33 & 28 & 29 \\
19 & 41 & 60 & 46 \\
\end{array}$ \\
\hline 03 & $\begin{array}{lllll}56 & 33 & 28 & 29 & {[146]} \\
19 & 41 & 60 & 46 & {[166]} \\
\end{array}$ & $\begin{array}{llll}23 & 25 & 28 & 25 \\
23 & 25 & 28 & 25 \\
\end{array}$ & $\begin{array}{l}117 \\
117\end{array}$ & $\begin{array}{llllll}6373 & 2932 & 3227 & 2791 & {[15323]} \\
1927 & 3885 & 7106 & 4651 & {[17569]}\end{array}$ & $\begin{array}{llll}51 & 26 & 18 & 22 \\
11 & 34 & 53 & 39 \\
\end{array}$ \\
\hline 04 & $\begin{array}{lllll}51 & 26 & 18 & 22 & {[117]} \\
11 & 34 & 53 & 39 & {[137]} \\
\end{array}$ & $\begin{array}{llll}14 & 33 & 23 & 29 \\
14 & 33 & 23 & 29 \\
\end{array}$ & $\begin{array}{l}115 \\
115\end{array}$ & $\begin{array}{llllll}5475 & 1803 & 2135 & 1977 & {[11890]} \\
1253 & 2741 & 6284 & 3785 & {[14063]} \\
\end{array}$ & $\begin{array}{llll}50 & 16 & 10 & 13 \\
07 & 24 & 48 & 31 \\
\end{array}$ \\
\hline 05 & $\begin{array}{cccccc}50 & 16 & 10 & 13 & {[} & 89] \\
07 & 24 & 48 & 31 & {[110]} \\
\end{array}$ & $\begin{array}{lll}08 & 19 & 12 \\
08 & 19 & 12 \\
\end{array}$ & $\begin{array}{l}71 \\
71 \\
\end{array}$ & 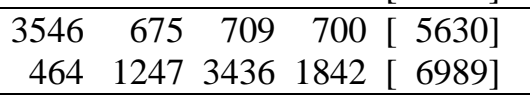 & $\begin{array}{llll}49 & 09 & 05 & 07 \\
04 & 17 & 45 & 26 \\
\end{array}$ \\
\hline 06 & $\begin{array}{lllll}49 & 09 & 05 & 07[70] \\
04 & 17 & 45 & 26[92] \\
\end{array}$ & $\begin{array}{lll}04 & 10 & 05 \\
04 & 10 & 05 \\
\end{array}$ & $\begin{array}{l}43 \\
43 \\
\end{array}$ & $\begin{array}{rrrrrr}2098 & 230 & 212 & 225 & {[} & 2765] \\
157 & 575 & 1939 & 935[ & {[3606]} \\
\end{array}$ & $\begin{array}{llll}48 & 05 & 03 & 04 \\
02 & 13 & 44 & 23 \\
\end{array}$ \\
\hline 07 & $\begin{array}{lllll}48 & 05 & 03 & 04[60] \\
02 & 13 & 44 & 23[ & {[82]}\end{array}$ & $\begin{array}{lll}02 & 05 & 03 \\
02 & 05 & 03\end{array}$ & $\begin{array}{l}30 \\
30\end{array}$ & 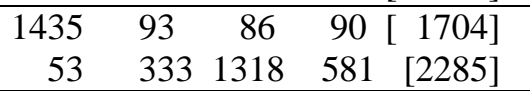 & $\begin{array}{llll}47 & 03 & 02 & 02 \\
01 & 11 & 43 & 22\end{array}$ \\
\hline 08 & $\begin{array}{llllll}47 & 03 & 02 & 02 & {[} & 54] \\
01 & 11 & 43 & 22 & {[} \\
\end{array}$ & $\begin{array}{lll}01 & 03 & 02 \\
01 & 03 & 02 \\
\end{array}$ & $\begin{array}{l}24 \\
24\end{array}$ & $\begin{array}{rrrr}44 & 45 & 36 & {[1251]} \\
236 & 030 & 450 & {[1737]} \\
\end{array}$ & $\begin{array}{llll}47 & 02 & 01 & 01 \\
01 & 10 & 42 & 21 \\
\end{array}$ \\
\hline 09 & $\begin{array}{lllll}47 & 02 & 01 & 01 & {[51]} \\
01 & 10 & 42 & 21 & {[74]} \\
\end{array}$ & $\begin{array}{lll}01 & 02 & 01 \\
01 & 02 & 01 \\
\end{array}$ & $\begin{array}{l}21 \\
21 \\
\end{array}$ & 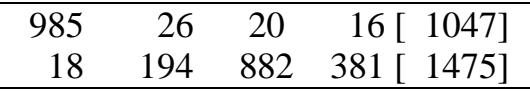 & $\begin{array}{llll}47 & 01 & 01 & 01 \\
01 & 09 & 42 & 21 \\
\end{array}$ \\
\hline 10 & $\begin{array}{llllll}47 & 01 & 01 & 01 & {[} & 50] \\
01 & 09 & 42 & 21[ & 73] \\
\end{array}$ & $\begin{array}{lll}01 & 01 & 01 \\
01 & 01 & 01\end{array}$ & $\begin{array}{l}20 \\
20\end{array}$ & 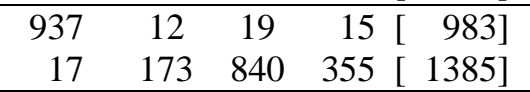 & $\begin{array}{llll}47 & 01 & 01 & 01 \\
01 & 09 & 42 & 21 \\
\end{array}$ \\
\hline 11 & $\begin{array}{lllll}47 & 01 & 01 & 01[ & 50] \\
01 & 09 & 42 & 21[ & {[73]} \\
\end{array}$ & $\begin{array}{lll}01 & 01 & 01 \\
01 & 01 & 01 \\
\end{array}$ & $\begin{array}{l}20 \\
20 \\
\end{array}$ & 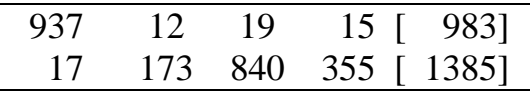 & $\begin{array}{llll}47 & 01 & 01 & 01 \\
01 & 09 & 42 & 21 \\
\end{array}$ \\
\hline
\end{tabular}

In the Table 2 given above, the results of cycle 10 and cycle 11 are the same. If this process continues into more cycles, the results will be the same as the results of cycle 10. Because of oversaturation situation some vehicles are still waiting on each lane in the last cycle (cycle 11).

Total number of vehicles at

Intersection 1

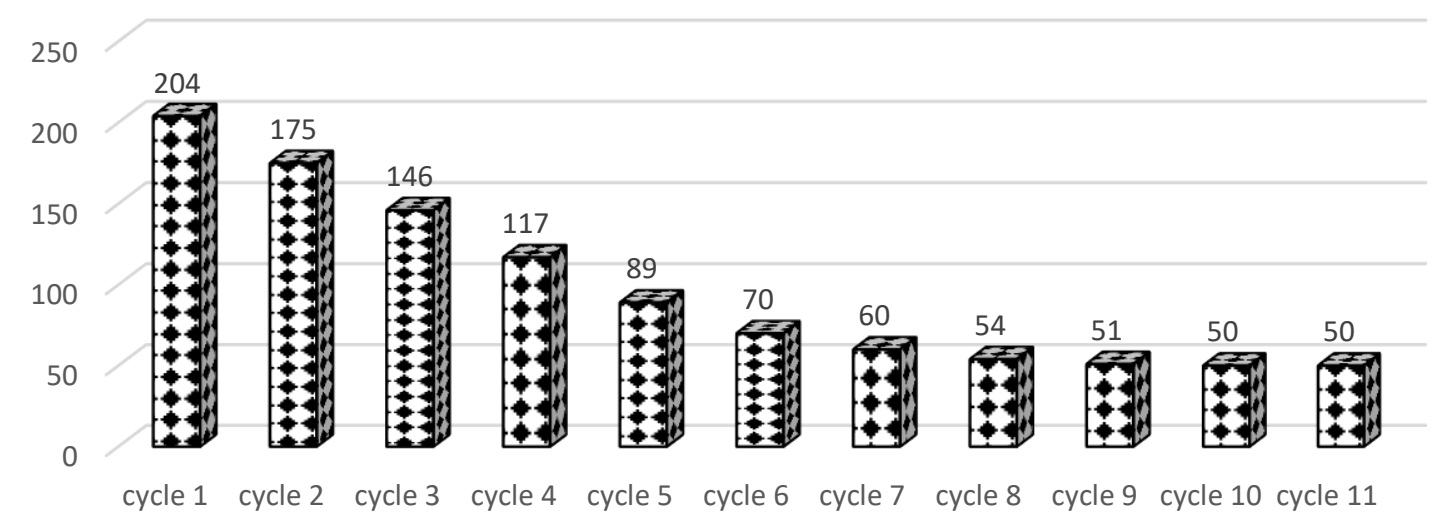

Figure 10: Total number of vehicles in each cycle at Intersection 1 of arterial network for Case II. 


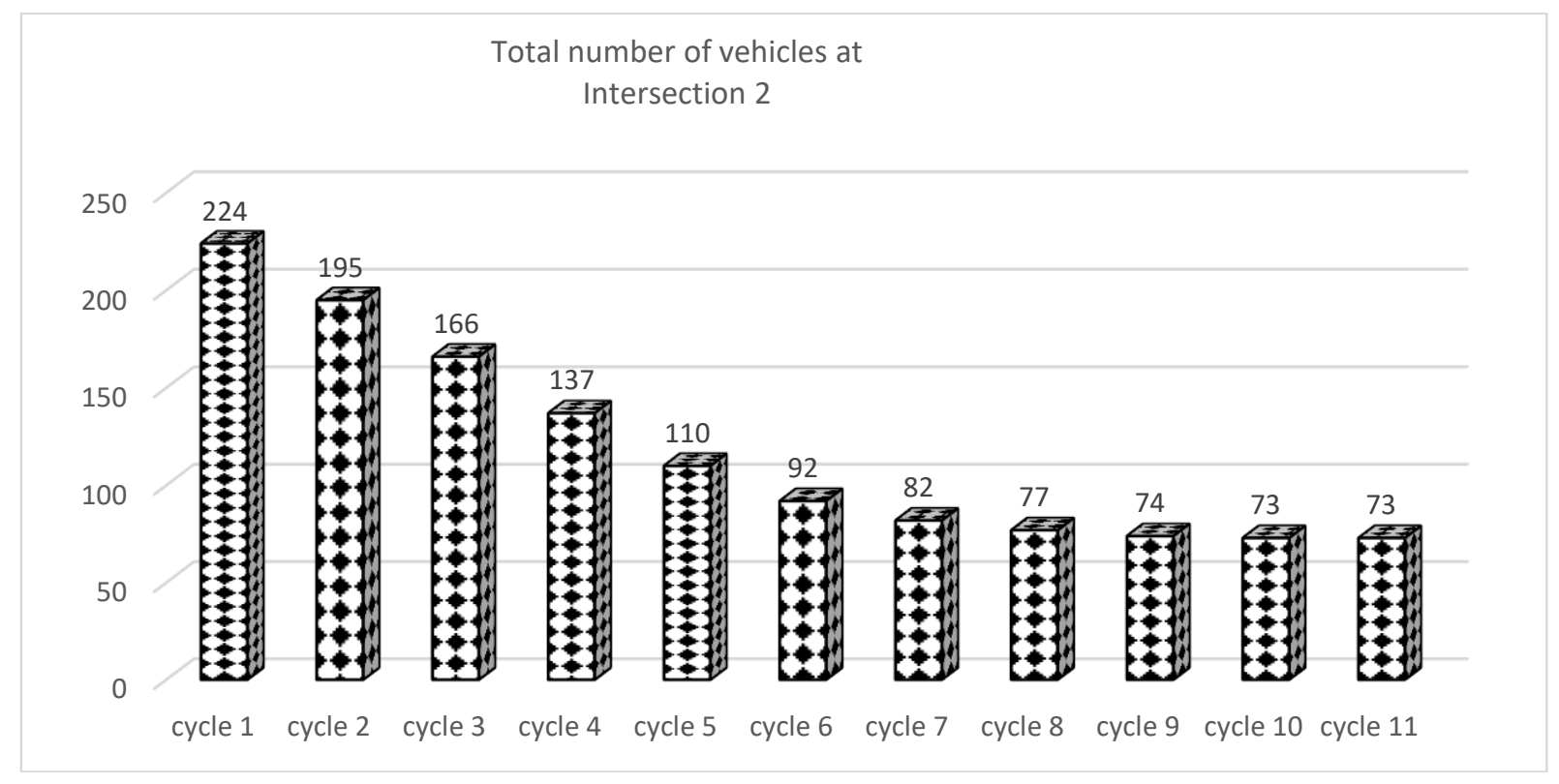

Figure 11: Total number of vehicles in each cycle at Intersection 2 of arterial network for Case II.

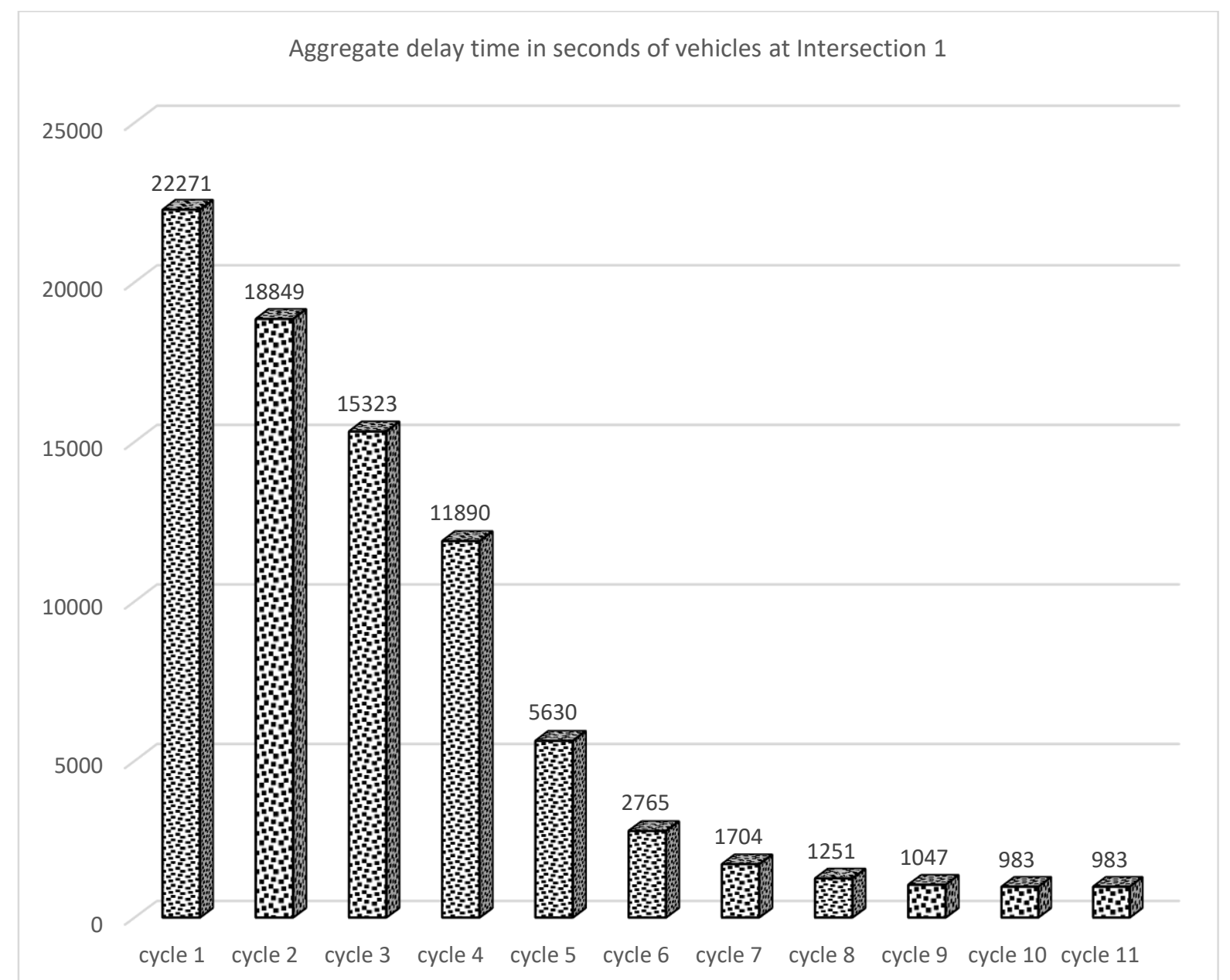

Figure 12: Aggregate delay time in each cycle at Intersection 1 of arterial network for Case II. 


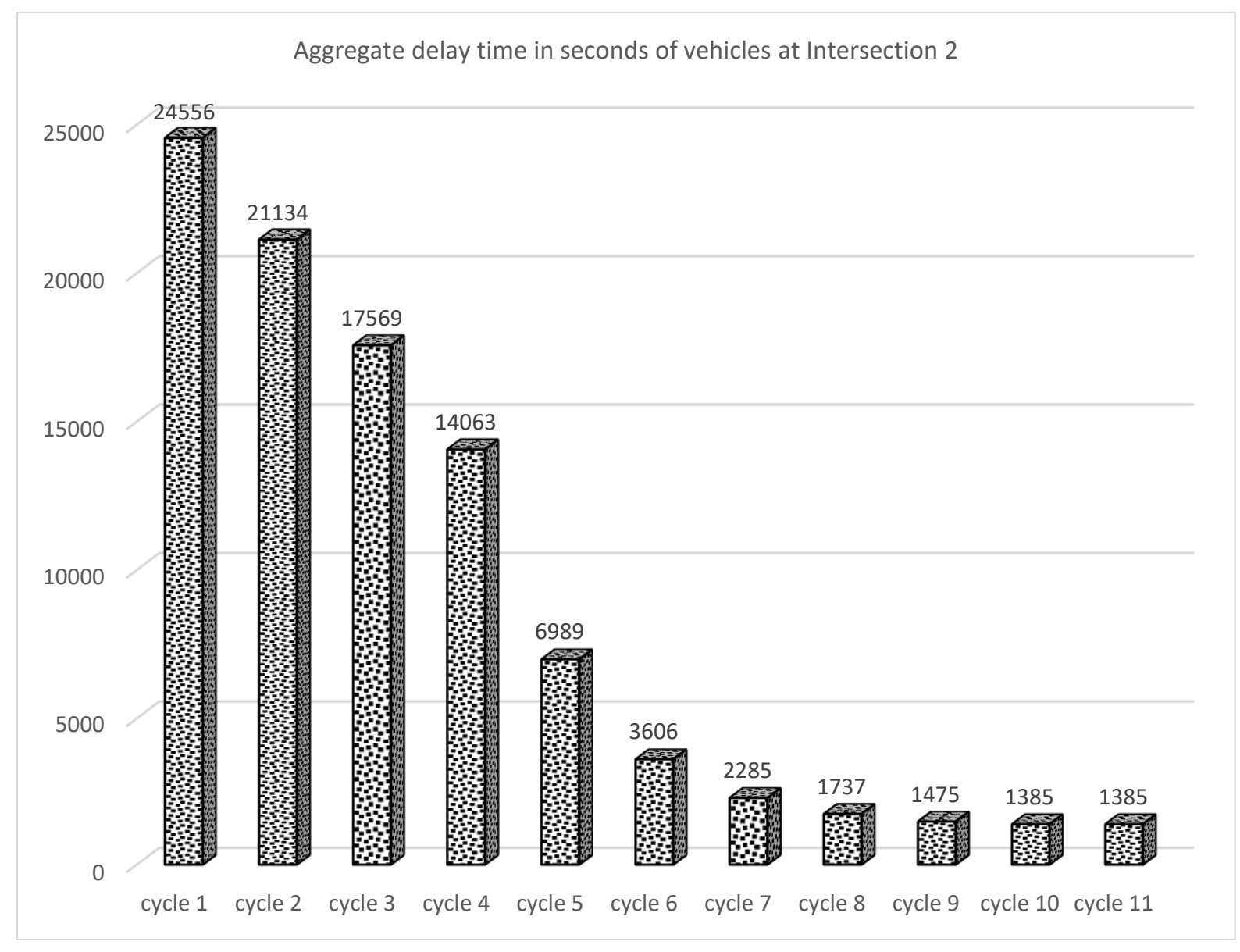

Figure 13: Aggregate delay time in each cycle at Intersection 2 of arterial network for Case II.

\section{Conclusion}

In this research paper, the developed models under control of two special oversaturation conditions along with some other control conditions are interfaced with the fmincon interior-point algorithm coded in MATLAB environment to create a real time optimized signal time control platform at a road consist of two intersections arterial network. Those models can be extended to more than two intersections arterial networks. In those models the incoming flow rate of vehicles on each lane is calculated during inter green-red-green signal transition time rather than cycle time. These models estimate green signal time of each signal for a particular cycle using the number of vehicles from camera reading at the beginning of that cycle. This process can continue cycle to cycle. But, we analyzed performance of vehicles only taking camera readings for the first cycle and for the rest of the other cycles, the number of vehicles at the intersection will be calculated using the results of the previous cycle. Those integrated models are applied to a hypothetical two intersections arterial network. The results of the estimation show that the proposed mathematical models produce better results than the other existing optimization models.

\section{References}

AKCELIK. R., (1980).Time dependent Expressions for Delay, Stop Rate and Queue Length at Traffic signals. Internal Report. AIR 367-1. Australian Road Research Board, Vermount South, Australia.

AhmetYazici, GangdoSeo, UmitOzguner, (2008). A model predictive control approach for decentralized traffic signal control, Proc. 17th World Congress, The international federation of Automatic Control, Seoul, Korea, July 6-11.

Aboudolas, K., Papageorgiou, M., Kouvelas, A., Kosmatopoulos, E. (2009). Store-and-forward based methods for the signal control problem in large-scale congested urban road networks. Transportation Research Part C: Emerging Technologies17(2), 163-174.

Gartner, N.H. (1983). OPAC: A Demand-Responsive Strategy for Traffic Signal Control. Transportation Research ecord 906, 75-81 
Henry J. J., Farges J. L., and J. Tuffal. (1983).The PRODYN Real Time Traffic Algorithm. In Proceedings of IFAC Control in Transportation Systems. Baden-Baden, Federal Republic of Germany, pp. 305-310.

Mrs.P.M.Daigavane, Dr.P.R.Bajaj, (2010). Real Time Vehicle Detection and Counting Method for Unsupervised Traffic Video on Highways, IJCSNS International Journal of Computer Science and IJCSNS International Journal of ComputerScience and Network Security, VOL.10 No.8, 2010.

Peng CHEN, Huan LIU, Hong-sheng QI, Fu-jian WANG. (2013). Analysis of delay variability at isolated signalized intersections, Journal of Zhejiang University-SCIENCE A (Applied Physics \& Engineering) 14(10):691704.

SCOOT User Guide: Version 4.2.Siemens Traffic Controls Ltd., Poole,Dorset, United Kingdom, 2003.

Tang-Hsien Chang, Jen-Ting Lin. (2000). Optimal signal timing for an oversaturated intersection. Transportation Research Part B: Elsevier Science Ltd.34, 471-491.

Yuanchang XIE. (2007). Development and Evaluation of an arterial adaptive Traffic Signal Control System Using Reinforcement Learning. Ph.D., Thesis, Office of Graduate Studies of Texas A\&M University.

Zhili Tian. 2002. Capacity Analysis of Traffic Actuated Intersections, Thesis MSc. Department of Civil and Environmental Engineering, Massachusetts Institute of Technology.

Zong Tian, Fred Ohence, Peifeng Hu. (2011). Arterial Performance Evaluation on an Adaptive Traffic Signal Control System, 6 th International Symposium on Highway Capacity and Quality of Service, Procedia Social and Behavioral Sciences 16 (2011) 230-239 June 28-July. 